\title{
Turbulent thermalization
}

\author{
Raphael Micha* \\ Theoretische Physik, ETH Zürich, CH-8093 Zürich, Switzerland \\ Igor I. Tkachev ${ }^{\dagger}$ \\ Department of Physics, Theory Division, CERN, CH-1211 Geneva 23, Switzerland \\ and Institute for Nuclear Research of the Russian Academy of Sciences, 117312, Moscow, Russia
}

(Received 11 March 2004; published 26 August 2004)

\begin{abstract}
We study, analytically and with lattice simulations, the decay of coherent field oscillations and the subsequent thermalization of the resulting stochastic classical wave field. The problem of reheating of the Universe after inflation constitutes our prime motivation and application of the results. We identify three different stages of these processes. During the initial stage of "parametric resonance," only a small fraction of the initial inflaton energy is transferred to fluctuations in the physically relevant case of sufficiently large couplings. A major fraction is transferred in the prompt regime of driven turbulence. The subsequent long stage of thermalization classifies as free turbulence. During the turbulent stages, the evolution of particle distribution functions is self-similar. We show that wave kinetic theory successfully describes the late stages of our lattice calculation. Our analytical results are general and give estimates of reheating time and temperature in terms of coupling constants and initial inflaton amplitude.
\end{abstract}

DOI: $10.1103 /$ PhysRevD.70.043538

PACS number(s): 98.80.Cq, 05.20.Dd, 11.10.Wx

\section{INTRODUCTION}

Field theoretical systems which are a long way from thermal equilibrium have been studied intensely in recent years. The particular problem of how and when such systems approach equilibrium stretches beyond obvious fundamental interest and finds many practical applications. In high-energy physics understanding of these processes is crucial for applications to heavy ion collisions and to cosmology of the early universe. The first topic gains further importance in light of the current and future experimental search for a quark-gluonplasma at RHIC and at the forthcoming LHC. The second application, our main interest in this paper, is related to the problem of reheating of the universe after cosmological inflation.

Inflation provides a solution to the flatness and the horizon problems of standard cosmology [1-3] and explains the generation of initial density perturbations- the seeds of galaxies and large-scale structure in our universe. During inflation the universe is in a vacuumlike state. At the end of inflation all energy density is stored in a Bose condensate, the coherently oscillating "inflaton" field. This state is highly unstable: parametric, tachyonic or strong nonadiabatic particle creation triggers a fast and explosive decay of the inflaton. This process, dubbed preheating [4-6], is currently well understood [7-14]. A generic feature is a strong and fast amplification of fluctuation fields at low momenta, which may lead to various interesting physical effects during and after preheating. These include nonthermal phase transitions [15-18] with possible formation of topological defects [19$23]$, creation of super-heavy particles [14,24], generation of high-frequency gravitational waves [25], etc.

\footnotetext{
*Electronic address: micha@itp.phys.ethz.ch

${ }^{\dagger}$ Electronic address: igor.tkachev@cern.ch
}

The explosive stage of inflaton decay ends when the rate of interactions of created fluctuations among themselves and with the inflaton becomes comparable to the inflaton decay rate [7-10]. The understanding of the subsequent stages of relaxation towards equilibrium, of the thermalization processes and the calculation of the final equilibrium temperature is important for various applications as it links the inflationary phase with that of standard cosmology. Among those one can list baryogenesis [14,26-29] and the problem of over-abundant gravitino production in supergravity models [30-35]. It determines the abundances of other relics, like super-heavy dark matter [36-39], or axino dark matter [40].

Knowledge of the reheating temperature is also important for fixing constraints on the inflationary model from cosmic microwave background (CMBR) anisotropy [41-44]. In some models cosmologically important curvature perturbations may be even generated during the process of thermalization [45-49]. Last but not least: the reheating temperature should be larger than about $1 \mathrm{GeV}$ to ensure that the standard Big Bang nucleosynthesis $[2,50]$ is not hampered.

There have been many efforts and successes in the understanding of the nonequilibrium dynamics and relaxation of field theories, see e.g. Refs. [51-66]. However, the leading asymptotic dynamics towards equilibrium remained rather less understood and developed.

The main problem for the theoretical understanding of reheating is that initially the occupation numbers are very large, of order of the inverse coupling constant. In addition, in many inflationary models the zero mode does not decay completely during preheating. Therefore, a simple perturbative approach is not justified. On the other hand, in this regime, a description in terms of classical field theory is valid [7], and the whole process (including preheating), can be studied by classical lattice simulations.

Recently, we employed this method to show $[67,68]$ that the classical reheating of a massless $\Phi^{4}$ theory in $3+1$ 
dimensions is characterized by a turbulent and self-similar evolution of distribution functions towards equilibrium. The shape of the spectra, as well as the self-similar dynamics, could be understood within the framework of wave kinetic theory. This made it possible to estimate reheating time and temperature, which turned out to coincide parametrically with the results of the simple perturbative approach.

Turbulence appears in a large variety of nonequilibriumphenomena in nature (see Refs. [69-71] for a general introduction). It was first discussed for fluids, in the regime of large Reynolds numbers (velocities), where viscosity is subdominant. Kolmogorov identified turbulence in this regime $[72,73]$ as a statistically scale invariant flow of spectral energy mediated by vortex interactions. The same dynamical structure may appear in systems of coupled waves, e.g. on fluid surfaces or for coupled fields in a plasma $[69,70,74]$. In this case the cascade is mediated by wave interactions and the phenomenon has been called wave turbulence.

If there exists an active (stationary) source of energy in momentum space, the turbulence is called driven (stationary). When the source is switched off after the stage of activity, the freely propagating energy cascade is often referred to as free turbulence. If the kinetic description is applicable, the energy cascade is called weak turbulence. Otherwise one is facing a strong turbulence.

One may expect that the concept of turbulence should be relevant for the problem of reheating [7,51] already on general grounds. Indeed, the source of energy, localized in the "infrared" is present initially. It is represented by the inflaton field in the problem at hands. To complete the argument, we note that as the final outcome of the evolution one should expect cascading of energy towards a significantly separated region of "ultraviolet," high momentum modes.

The goal of the current paper is twofold. First, we want to apply the wave kinetic theory of turbulence to the problem of the Universe reheating after inflation. We derive general formulas for the spectra of turbulent distributions and for the self-similar evolution towards equilibrium. This enables us to give asymptotic estimates of reheating time and temperature in Minkowski space as well as in the Friedmann universe.

Second, we want to test and confront these ideas to numerical lattice calculations. For our numerical integrations we have chosen the simplest "chaotic" inflationary model [75]. While the initial "preheating" stage in other inflationary models, e.g. in hybrid inflation [76] may exhibit important differences $[12,13,77]$ with this model, we expect the subsequent turbulent stages to be more universal.

We start lattice integration from "vacuum" initial conditions for fluctuations in a background of classical oscillating inflaton field. We observe the initial parametric resonance stage when the energy in fluctuations is growing exponentially with time. This stage terminates when re-scattering of waves out of the resonance band becomes important [7,9]. In the physically relevant case of sufficiently large couplings this happens rather early, when only a small fraction of initial inflaton energy is transferred to fluctuations [9-11]. At this point a state of stationary turbulence should be established that is driven by the zero mode. On general grounds, it can be deduced that during this stage the energy in fluctuations should grow linearly with time. This behavior is confirmed by the results of our numerical simulations. The stage of stationary turbulence should terminate when the energy left out in the zero mode becomes smaller than the energy stored in created "particles." From this moment of time, the transport of energy from the source is negligible and we observe free turbulence with self-similar evolution of particle distributions towards thermal equilibrium.

The first stage of driven turbulence is prompt and gives the main mechanism by which energy is drawn out of the zero mode, e.g. out of the inflaton field. The identification of this constitutes one of the new results of the present paper, as opposed to the common opinion that the main mechanism is a "parametric resonance." The second stage of free turbulence is very long and can be analytically described as selfsimilar evolution. This is another new result and diffuses some existing claims and hopes that "parametric resonance" may bring a system to thermal equilibrium on a very short time scale.

Overall, the kinetic description and the results of lattice simulations are in rather good agreement with each other. This indicates that the regime of weak wave turbulence may be already achieved on the lattice.

The paper is organized as follows. In Sec. II we review the results of our numerical simulation of reheating in the simplest $\lambda \Phi^{4}$ model to get familiar with concepts, problems and the typical dynamical behavior of the systems of interest. In Secs. III and IV we apply the theory of wave turbulence to the problem of reheating in general. In Sec. V we present our numerical simulations. In Sec. VI we compare lattice results with the kinetic approach and discuss the applicability of the latter. In Sec. VII we discuss some physical applications of our results, in particular the thermalization in the self-similar regime. In Appendix A we give the details of our numerical procedure. In Appendix B we review the derivation of the kinetic equation for a system of weakly interacting classical waves.

\section{SIMPLEST MODEL OF REHEATING: NUMERICAL RESULTS}

We start with a presentation of our numerical results for the inflaton decay and the subsequent equilibration of the decay products in a simple $\lambda \Phi^{4}$ model. The results were already briefly reported in Ref. [67]. The numerical procedure itself is described in Appendix A. At the end of the section we will discuss some expected differences with more complicated models. This order of presentation allows us to introduce the typical behavior in the systems under consideration and to formulate concepts and problems. This will be useful in the discussion of the general theory of turbulent thermalization, which we carry out in the following section. Further numerical results, obtained for the simplest $\lambda \Phi^{4}$ model, and numerical results obtained for more complicated multifield systems, will be presented in Secs. V and VI.

\section{A. Results for the $\Phi^{4}$ model}

In this simple model, the field $\Phi$ is the only dynamical variable. Its initial homogeneous mode drives inflation, while 
development and growth of fluctuations on sub-horizon scales at the end of inflation can be viewed as a simple model of reheating. Inflation ends when the motion of the homogeneous mode of the field changes from the regime of "slow roll" to the regime of oscillations. It is convenient to work in conformal coordinates where the metric takes the form

$$
d s^{2}=a(\eta)^{2}\left(d \eta^{2}-d x^{2}\right)
$$

We choose the case of a massless field, where the equation of motion for the rescaled field $\varphi \equiv \Phi a$ after inflation approaches the flat space-time equation

$$
\square \varphi+\lambda \varphi^{3}=0 .
$$

This point deserves clarification, since in the Friedmann universe the field equations, in general, contain a linear term proportional to $-\ddot{a} / a$. However, this term is unimportant at preheating and subsequent stages, see e.g. Refs. [7,9] where it was included self-consistently in lattice integrations. Indeed, during the initial parametric resonance stage a coherently oscillating field does induce small oscillations of the scale factor on top of radiationlike dominated expansion, however, the corresponding resonance parameter is small and does not lead to significant effects. With the end of parametric resonance stage and the subsequent chaotization, the equation of state rapidly approaches that of radiation (for massless fields $p=\rho / 3$ regardless of distribution of particles over momenta, and, consequently, this is true for highly nonequilibrium states as well). The solution of Friedmann equations in this case is $a(\eta) \propto \eta$, or $\ddot{a}=0$. The term proportional to $-\ddot{a} / a$ is not important for the dynamics of reheating and thermalization, and we neglect it in what follows.

All results obtained in the model Eq. (2) are equally applicable to the reheating of the Universe after inflation and to modeling of other processes of thermalization in relativistic systems, say, after heavy ion collisions.

The homogeneous component of the field, which corresponds to the zero momentum in the Fourier decomposition, $\varphi_{0}(\eta) \equiv\langle\varphi\rangle$, is usually referred to as the "zero mode." It is convenient to make a rescaling of the field, $\phi \equiv \varphi / \varphi_{0}\left(\eta_{0}\right)$, and of the space-time coordinates, $x^{\mu} \rightarrow \sqrt{\lambda} \varphi_{0}\left(\eta_{0}\right) x^{\mu}$. Here, $\eta_{0}$ corresponds to the initial moment of time (end of inflation). In what follows dimensionless time is still denoted as $\eta$. With this rescaling, the initial condition for the zero-mode oscillations is $\phi_{0}\left(\eta_{0}\right)=1$, and the equation of motion takes the simple parameter free form

$$
\square \phi+\phi^{3}=0
$$

All model dependence on the coupling constant $\lambda$ and on the initial amplitude of the field oscillations is encoded now in the initial conditions for the small (vacuum) fluctuations of the field with nonzero momenta (see Ref. [7] and Appendix A). The physical normalization of the inflationary model corresponds to a dimensionful initial amplitude of $\varphi_{0}\left(\eta_{0}\right)$ $\approx 0.3 M_{\mathrm{Pl}}$ and a coupling constant $\lambda \sim 10^{-13}$ [1]. The re-

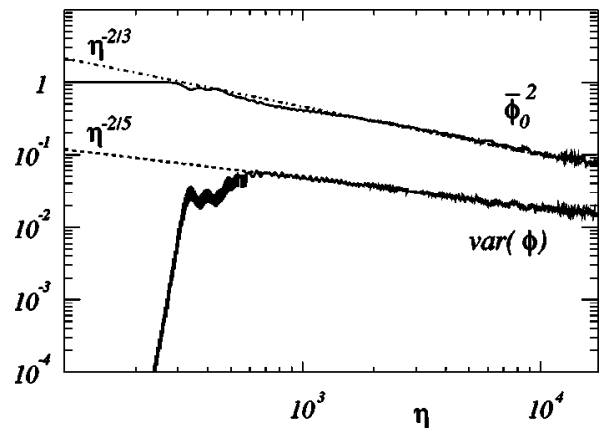

FIG. 1. Squared amplitude of the zero-mode oscillations, $\bar{\phi}_{0}^{2}$, and variance of the field fluctuations as functions of time $\eta$.

parametrization property of the system allows to choose a larger value of $\lambda$ for numerical simulations. We have used $\lambda=10^{-8}$.

Various quantities can be measured in a lattice calculation and monitored as functions of time. Here we will discuss the zero mode, $\phi_{0} \equiv\langle\phi\rangle$, the variance, $\operatorname{var}(\phi) \equiv\left\langle\phi^{2}\right\rangle-\phi_{0}^{2}$ and "particle occupation" numbers. For definitions see Appendix A.

We begin the discussion of our numerical results with the evolution of the zero mode and the variance of the field, which are shown in Fig. 1. The zero mode is a rapidly oscillating function on the time scale of our lattice calculation. In Fig. 1 we show the amplitude of oscillations, $\bar{\phi}_{0}$, as a function of time.

The initial fast transfer of the zero-mode energy into fluctuations during preheating (up to $\eta \sim 300$ ) is followed by a long and slow relaxation phase. In this late time regime the amplitude of the zero mode oscillations decreases according to $\sim \eta^{-z}$ with $z \approx 1 / 3$, the variance of the field (averaged over high-frequency oscillations) drops according to $\sim \eta^{-v}$ with $v \approx 2 / 5$. In addition, we find that in this regime the zero mode is in a nontrivial dynamical equilibrium with the bath of highly occupied modes: when the zero mode is artificially removed, it is recreated on a short time scale (Bose condensation).

A detailed analytical discussion of the initial linear stage of the parametric resonance in this model can be found e.g. in Refs. [78-80]. During this stage the occupation numbers grow exponentially with time in a narrow band of resonance momenta. Figure 2 shows the occupation numbers at differ-

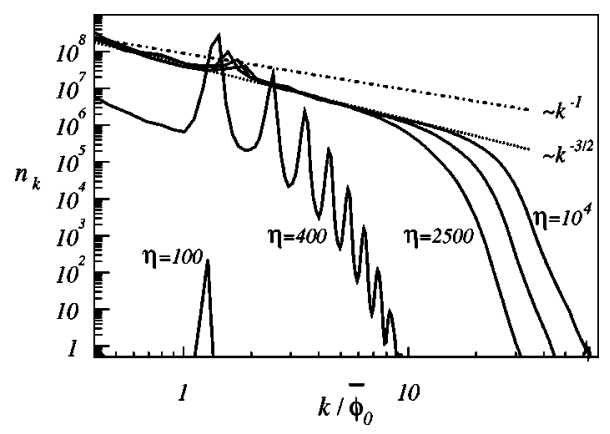

FIG. 2. Occupation numbers as function of $k / \bar{\phi}_{0}$ at $\eta=100,400,2500,5000,10000$. 
ent moments of time. The displayed spectrum at time $\eta$ $=100$ corresponds to the stage of parametric resonance. The resonance peak is located at the theoretically predicted value of $k_{\text {res. }} \sim 1.27$ [79]. At later time, the growth of the resonance peak is stopped by re-scattering of particles out of the resonance band, which leads to a broadening of the occupied region and to the appearance of multiple peaks [7] of comparable width, see spectrum at $\eta=400$. This structure fits estimates for the development of turbulence in the presence of a narrow width source located at a finite $k$, see Ref. [69]. At even later times the spectra have become smooth because of re-scattering, and only the first peak is still visible as a small bump. With time, its position moves towards smaller momenta, reflecting the change in the effective frequency of inflaton oscillations. However, if the particle momenta are rescaled by the current amplitude $\bar{\phi}_{0}$ of the zero mode oscillations, as in Fig. 2, the position of the resonance is approximately unchanged. Particles with small momenta are distributed according to a power law, which at larger momenta is bounded by a cutoff. The position of this cutoff moves with time to the "ultraviolet." This reflects a general tendency of the system to thermal equilibrium. Indeed, in a state of thermal equilibrium the energy of the system should be concentrated at much higher wave numbers compared to the resonance momenta. On the other hand, energy is inputted into the system of particles in the region of $k$ near the resonance peak. Therefore, we have a continuous flux of energy across momentum space, from low to high momenta.

This stage of evolution ( $\eta>1500)$ has the following characteristic features:

(i) The system overall is statistically close to a Gaussian distribution of field amplitudes and conjugated momenta $[58,67]$.

(ii) The spectra in the dynamically important region can be described by a power law, $k^{-s}$ with $s \approx 3 / 2$. We see that the system is not in a thermal equilibrium which would correspond to $s=1$. Rather, the exponent of particle distributions in the power law region corresponds to Kolmogorov turbulence [67].

(iii) The power law is followed by a cutoff at higher $k$. Energy accumulated in particles is concentrated in the region were the cutoff starts. Its position is monotonously growing toward the ultraviolet, reflecting the evolution towards thermal equilibrium.

(iv) This motion can be described as a self-similar evolution [67]

$$
n(k, \tau)=\tau^{-q} n_{0}\left(k \tau^{-p}\right),
$$

where $\tau \equiv \eta / \eta_{c}$ and $\eta_{c}$ is some (arbitrary) late-time moment. The best numerical fit corresponds to $q \approx 3.5 p$ and $p \approx 1 / 5$, and is presented in Fig. 3. The value of the exponent $p$ is of prime interest since it determines the rate with which system approaches equilibrium.

The first and the second point in this list facilitate the use of wave kinetic theory, see e.g. Refs. $[69,81]$. However, a straightforward application is difficult and may be even inappropriate, at least at the early re-scattering stages, because of the following:

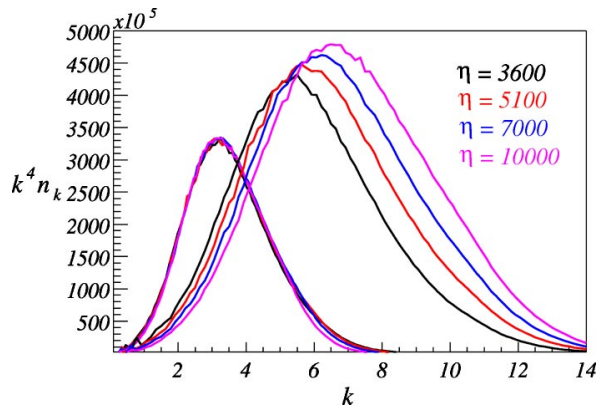

FIG. 3. On the right hand side we plot the wave energy per decade found in lattice integration at $\eta=3600,5100,7000,10000$. On the left hand side are the same graphs transformed according to the relation inverse to Eq. (4).

(i) The zero mode does not decay completely. It may induce "anomalous" terms in the collision integral, which are absent in the usual kinetic description.

(ii) The occupation numbers are large initially, of order of the inverse coupling constant, $n_{k} \sim 1 / \lambda$, see Fig. 2 . Therefore, in addition to lowest order collisions (e.g. scattering of two particles into two particles with different momenta), multiparticle collisions may be dynamically important as well.

Therefore, precise lattice calculations are needed. On the other hand, they have a limited dynamical range in momenta and in time, and one has to switch to a kinetic approach at some later stage. To determine when (and if) this is possible, the results obtained with the use of a simple kinetic approach should be confronted with the lattice results.

In the following sections we will develop and apply the theory of weak wave turbulence to the models of the type integrated on the lattice. In particular, we will calculate all universal scaling exponents and show that they are in agreement with lattice results. At "early" times the dynamics of the model described above is driven by $m$-particle interactions with $m=3$. Wave turbulence theory gives for scaling exponents in $d=3$ spatial dimensions:

$$
\begin{aligned}
& p=1 /(2 m-1), \\
& s=d-m /(m-1), \\
& v=2 /(2 m-1), \\
& z=1 /[d(m-1)-m] .
\end{aligned}
$$

\section{B. Expected differences in more complicated models}

The flux of energy over momentum space, which is necessarily present in problems like reheating and thermalization after inflation, signifies that we should observe a turbulent state during the thermalization stage and that the theory of turbulence applies. In a simple $\lambda \phi^{4}$ model the stage of preheating (i.e. parametric resonance) ends when roughly half of the inflaton energy is transferred to particles. Indeed, Fig. 1 shows that the amplitude of the zero mode, which is a source of energy for the turbulence problem, starts to de- 
crease already at the end of the parametric resonance stage. In such system we expect the free turbulence regime to follow the preheating stage.

In more complicated systems, which involve other fields coupled to the inflaton, say, some field $\chi$, parametric resonance may end when the fraction of energy transferred to the $\chi$ excitations is still negligible compared to the energy stored in the inflaton zero mode. Indeed, parametric resonance ends when the rate of re-scattering of particles out of the resonance band became comparable to the resonant production rate and the maximal value of the variance of $\chi$ excitations achieved at the end of the resonance stage is $\sim 1 / g^{2}$, where $g^{2}$ is either the coupling of $\chi$ to the inflaton, or self-coupling of $\chi$ (viz., the largest of these two). We expect that in this case turbulent transport will develop when the amplitude of the inflaton zero mode is still unchanging. This means that the transfer of zero mode energy into $\chi$-field should occur in the regime of stationary turbulence. Only when the amount of energy in the zero mode becomes subdominant we should expect a transition to the regime of free turbulence. This is an important difference to the simple $\phi^{4}$ model. In particular, the distribution functions move much faster into the ultraviolet in this regime, $p=(m-1) /(2 m-1)$. We will see that the regime of stationary turbulence is indeed present in two field models, see Sec. V.

\section{THERMALIZATION IN THE WAVE KINETIC REGIME: GENERAL THEORY}

\section{A. Turbulent reheating: A motivation}

Kolmogorov's turbulence is characterized by a stationary transport of some conserved quantity between different scales in momentum (Fourier) space [72,73]. In the following, we will restrict ourselves to systems with spatially isotropic and homogeneous correlation functions, which applies to the cosmological conditions after inflation. Turbulence usually appears when a source of energy or particles is present and is localized in some momentum region $k_{\text {in }}$. In addition to the source exists a "sink" localized at $k_{\text {out }}$. When both, source and sink are stationary, it is natural to expect the eventual development of a stationary state with scale independent transport of the conserved quantity through momentum space. Indeed, energy or particle number cannot accumulate between $k_{\text {in }}$ and $k_{\text {out }}$ and should flow from one scale to the other.

This is a system-independent formulation of Kolmogorov's concept of turbulence, which he formulated in the context of hydrodynamical systems [72,73]. Zakharov applied it to systems of coupled waves [74] in the regime of kinetic wave interactions. His approach is based on his derivation of the wave kinetic equations (see e.g. Refs. $[69,74,81]$ ) and is well suited to studies of turbulence in classical field theories. We will adopt it here.

The physical scenario of reheating after inflation shares basic ingredients with that of turbulence: there exists a localized source of energy - the coherently oscillating inflaton zero mode-pumping energy into the system of particles at Fourier wave numbers $k_{\text {in }} \sim k_{\text {res }}$. The mechanism behind this pumping can be parametric resonance, tachyonic amplifica- tion, etc. Like in the turbulent scenario there do not exist other intermediate scales (wave numbers), where energy is infused, accumulated, or dissipated. Thus, it seems likely that the eventual dynamics of reheating - after the explosive regime of preheating has ended-is close to that of Kolmogorov's turbulence.

However, in the description of reheating appear some differences to stationary turbulence, since:

(i) A sink does not exist.

(ii) The source (i.e. the amplitude of inflaton zero-mode oscillations and therefore interaction rates) can be essentially time dependent on relevant time scales.

(iii) Neither source nor sink exist when the inflaton has completely decayed.

In the first case, we expect that the stationary turbulent flux of energy still will be established in some "inertial" range $k_{\text {in }}<k<k_{\text {out }}$. Particle distributions in this range of momenta should not be significantly different compared to the case with a stationary sink. Indeed, in the typical turbulent problem the energy dissipates (e.g. into heat) after entering the region $k \gg k_{\text {out }}$. For problems relevant to thermalization after inflation, instead of dissipation the transported energy is used to populate high momentum modes at $k \gg k_{\text {out }}$. If the transport is reasonably "local" in momentum space, the flux of energy through the inertial range should not be influenced much by processes which involve $k>k_{\text {out }}$. Energy may dissipate at $k_{\text {out }}$ or continue the flow to even higher momenta, but regardless of this, we should expect the same distribution of particles in the inertial range. However, in the latter case we can expect that the value of $k_{\text {out }}$ increases, and since the flux of energy is constant throughout inertial range, the total energy of a system without a sink has to grow linearly with time,

$$
E(t) \propto t
$$

This is a simple consequence of the stationarity of turbulence in the inertial range, and can be used as its signature.

A time dependent source (second point above) changes the picture somewhat, since stationary states are not likely to develop even in a finite range of $k$. However, a weak time dependence should still allow for a close-to-stationary and close-to-turbulent evolution. Moreover, even if the source eventually does not exists, particle distributions in the inertial range as functions of momenta can still be close to turbulent power laws. Indeed, stationary turbulent distributions can be found as zeros of the collision integral [69]. In the nonstationary case the collision integral is nonzero, but should approach a minimal value in the inertial range which may result in the same shape of particle distributions there.

\section{B. Wave turbulence by scaling analysis}

The dynamics of coupled waves close to a stationary state can be described by a wave kinetic equation (see e.g. Refs. $[69,74,82])$ :

$$
\dot{n}_{k}=I_{k}[n] .
$$

Here the function $n_{k}$, usually called occupation number or wave action, describes the average volume of phase space 
occupied by the oscillations of a single mode with a wave number $k$. Its evolution is a result of resonant wave interactions, the effect of which is described by the collision integral $I_{k}[n]$. The collision integral is a function of the "external" momentum $k$ and a functional of the distribution function $n$, which is reflected in the notations we use. When we do not need to stress the functional dependence, we will also write $I_{k}$ as $I(k)$. The collision integral for the case of interest, Eq. (2), is explicitly derived in Appendix B.

Before we proceed, let us remind the general structure of the collision integrals using as illustration the scattering of two particles into two particles, which will be referred to as a 4-particle process. This will also allow us to introduce the necessary notations. In all cases we will write the collision integral as

$$
I_{k}[n]=\int d \Omega\left(k, q_{i}\right) F\left(k, q_{i}\right) .
$$

This form separates the contributions which are due to the (fixed) particle model, $d \Omega\left(k, q_{i}\right)$, from those which are due to the (evolving) particle distribution functions, $F\left(k, q_{i}\right)$. Here $k$ is the external momentum and $q_{i}$ refer to momenta over which the integration is carried out. If $m$ particles participate in the collision, $i$ takes values from 1 to $m-1$. E.g. when 2 particles scatter into 2 particles, $m=4$ and there are 3 internal momenta over which we integrate, $q_{1}, q_{2}$ and $q_{3}$, namely

$$
d \Omega\left(k, q_{i}\right)=\frac{(2 \pi)^{4}|M|^{2}}{2 \omega_{k}} \delta^{4}\left(k_{\mu}, q_{i \mu}\right) \prod_{i=1}^{3} \frac{d^{3} q_{i}}{2 \omega_{i}(2 \pi)^{3}} .
$$

$d \Omega$ contains the usual energy-momentum conservation $\delta$ functions, which we have denoted as $\delta^{4}\left(k_{\mu}, q_{i \mu}\right)$, the "matrix element" squared, $|M|^{2}$, of the corresponding process (which is a function of $k$ and $q_{i}$ ) and the integration measure over momentum space. Here, $k_{0}=\omega_{k}=\omega(k)$ and $\omega_{i}$ $=\omega\left(q_{i}\right)$ refers to the particle energy.

When quantum effects are accounted for, the function $F$ in our example is given by

$F\left(k, q_{i}\right)=\left(1+n_{k}\right)\left(1+n_{q_{1}}\right) n_{q_{2}} n_{q_{3}}-n_{k} n_{q_{1}}\left(1+n_{q_{2}}\right)\left(1+n_{q_{3}}\right)$.

In the limit $n \gg 1$ terms $O\left(n^{2}\right)$ can be neglected and $F$ is a sum of terms $O\left(n^{3}\right)$,

$$
F\left(k, q_{i}\right)=\left(n_{k}+n_{q_{1}}\right) n_{q_{2}} n_{q_{3}}-n_{k} n_{q_{1}}\left(n_{q_{2}}+n_{q_{3}}\right) .
$$

The limit $n \gg 1$ corresponds to interaction of classical waves and expression Eq. (10) is also explicitly derived in Appen$\operatorname{dix}$ B. This illustrates a general rule: in the classical limit and for interaction of $\mathrm{m}$ waves the function $F$ is a sum of terms $O\left(n^{m-1}\right)$ with appropriate permutations of signs and indices. In other words, in this limit $F$ is a homogeneous function with respect to multiplication of each occupation number by $\zeta$

$$
F(\zeta n)=\zeta^{m-1} F(\zeta n)
$$

This property is extremely important in our subsequent analysis. When quantum effects become important (i.e. when one should properly write $[1+n]$ in F), the classical turbulence and/or self-similar evolution stops. At that moment particle distributions relax to usual Bose-Einstein functions. We will not be concerned here with the (presumably relatively short) relaxation period from the classical to the quantum regime, but will study in detail the turbulent evolution in the regime of classical waves.

This gives us sufficient notational details to proceed with the discussion of turbulence. We restrict it to systems which are isotropic and homogeneous in configuration space, when occupation numbers (as well as all other parameters which enter the collision integral) depend on the modulus of momenta only. We consider the classical limit in the function $F$ with general $\mathrm{m}$-particle interaction, in case of which Eq. (11) holds. To keep the discussion general, in the rest of this section we will consider the case of $(d+1)$ dimensional space time.

Often a collision integral conserves one or several quantities. We restrict ourselves to energy density

$$
\rho=\int \frac{d^{d} k}{(2 \pi)^{d}} \omega_{k} n_{k},
$$

which is conserved when the expansion of the Universe can be neglected or "rotated" away, and particle density

$$
n=\int \frac{d^{d} k}{(2 \pi)^{d}} n_{k}
$$

which corresponds to conserved charges, e.g. baryon number.

Conservation of $n$ or $\rho$ can be expressed as a continuity equation in Fourier space, e.g.

$$
\partial_{t}\left(\omega_{k} n_{k}\right)+\nabla_{k} \cdot j_{k}=0 .
$$

Here and in what follows we will write the explicit relation for energy conservation, the case of conserved charges can be easily obtained by a formal substitution $\omega_{k}=1$. In the isotropic case only the radial component of the flux density, $j_{k}$, is nonvanishing and we get for the energy flux, $S^{\rho}(p)$, trough the sphere of radius $p$,

$$
\begin{aligned}
(2 \pi)^{d} \cdot S^{\rho}(p) & =-\int^{p} d^{d} k \omega_{k} \dot{n}_{k} \\
& =-\frac{\pi^{d / 2}}{\Gamma\left(1+\frac{d}{2}\right)} \int^{p} d k k^{d-1} \omega_{k} I_{k}[n],
\end{aligned}
$$

In Eq. (15) the factor in front of the integral is the area of the d-dimensional unit sphere. In case of stationary turbulence this flux should be scale independent, i.e. integral Eq. (15) should not depend upon its integration limit $p$. This is possible if the collision integral equals zero. One can explicitly look for solutions $I_{k}[n]=0$, see e.g. Ref. [69]. Such solutions correspond to stationary turbulence and exist with non- 
trivial boundary conditions (source and sink), in addition to the Rayleigh-Jeans law of classical equilibrium. Here we adopt an alternative and somewhat simpler approach of Ref. [83] to determine the turbulent solutions.

Following Ref. [83] we consider states for which the collision integral has certain scaling properties under $\xi$ rescaling of the external momentum $k$,

$$
I_{\xi k}[n]=\xi^{-\nu} I_{k}[n] .
$$

To simplify notations we assume that all momenta were made dimensionless by rescaling with some typical momentum scale, without explicitly writing this. The special choice $\xi=k^{-1}$ allows us to find the $k$ dependence of the collision integral, $I_{k}[n]=k^{-\nu} I_{1}[n]$. Let us additionally assume that the dispersion law is a homogeneous function as well,

$$
\omega(\xi k)=\xi^{\alpha} \omega(k) .
$$

Relations (16) and (17) should hold in some region of momenta where we expect turbulent behavior. Integrating Eq. (15) we find

$$
S(p) \propto-p^{d+\alpha-\nu} \frac{I_{1}(\nu)}{d+\alpha-\nu} .
$$

Here we indicated explicitly that the collision integral in the turbulent state with scaling behavior Eq. (16) depends on the exponent $\nu$. We find that the flux is scale invariant, if

$$
\nu=d+\alpha .
$$

This condition defines the turbulent exponents which we will specify in detail below. Note that this implies the existence of the limit

$$
\lim _{\nu \rightarrow d+\alpha} \frac{I_{1}(\nu)}{d+\alpha-\nu}=\mathrm{const} \neq 0,
$$

as a sufficient condition for the existence of a stationary turbulent solution: if the collision integral has a zero of first degree at $\nu=d+\alpha$, the turbulent flux is scale invariant and finite.

In what follows, we consider particle models for which $d \Omega$ is a homogeneous function of all momenta

$$
d \Omega\left(\xi k, \xi q_{i}\right)=\xi^{\mu} d \Omega\left(k, q_{i}\right) .
$$

Rescaling of the external momentum $k$ by $\xi$ gives

$$
I_{\xi k}=\xi^{\mu} \int d \Omega\left(k, q_{i}\right) F\left(\xi k, \xi q_{i}\right),
$$

since integration over every $q_{i}$ is from 0 to $\infty$. We will exploit this relation in two ways:

(i) Often the evolution of distribution functions involves rescaling of their momenta, see Sec. III C. If this is the case, the collision integral as a function of time can be found with the help of

$$
\int d \Omega\left(k, q_{i}\right) F\left(\xi k, \xi q_{i}\right)=\xi^{-\mu} I_{\xi k}
$$

(ii) Let us assume that the particle distribution functions are power laws in the momenta,

$$
n(q) \propto q^{-s} .
$$

This leads to the following scaling of F:

$$
F\left(\xi k, \xi q_{i}\right)=\xi^{-s(m-1)} F\left(k, q_{i}\right) .
$$

Combining this with Eq. (22) we find $I_{\xi k}=\xi^{\mu-s(m-1)} I_{k}$. A comparison with Eqs. (16) and (19) leads us to the exponent $s$ which defines the scaling of particle distribution functions in a turbulent state with constant energy transport (we will call this energy cascade for brevity)

$$
s=\frac{d+\alpha+\mu}{m-1} .
$$

Turbulence with constant transport of particle number (similarly, we will call this state particle cascade) can be found at this point by the formal substitution $\omega=1$, i.e. $\alpha=0$ and

$$
s=\frac{d+\mu}{m-1} .
$$

Note that doing this substitution at later stages would be confusing since the explicit expression for $\mu$ also contains $\alpha$. Note also that on turbulent states $I[n]=0$, therefore, transport of all quantities except energy is zero for energy cascade. For particle cascade, which describes Bose condensation $[84,85]$, the transport of energy is zero.

The reader should bear in mind that only those solutions that describe the transport of energy towards the ultraviolet, $S^{\rho}>0$, are relevant for the problem of thermalization after inflation. The sign of fluxes for stationary turbulence of three- and four-wave collision integrals was found in Ref. [83],

$$
\operatorname{sgn} S^{\rho}=\operatorname{sgn}[\alpha s(s-\alpha)] .
$$

In thermal equilibrium $n \propto \omega^{-1}$, i.e. $s=\alpha$. Therefore, energy turbulence is directed towards the ultraviolet if the distribution function with increasing momenta falls off faster than in equilibrium, $s>\alpha$. As we will see, in the $\lambda \phi^{4}$ model this condition holds in $d=3$, but is violated at $d \leqslant 2$. Therefore, we believe that simulations of the thermalization in this model at $d<3$, see e.g. Refs. [53,57,59,64], may not reflect all aspects of the physical problem of reheating after inflation correctly.

\section{Self-similar evolution}

In an analytical approach to non-stationary situations (e.g. when describing free turbulence) it is usually assumed that the evolution is self-similar $[86,87]$. As we have shown, the evolution is self-similar, indeed, at late times in our numerical integration of the $\phi^{4}$ model, see Sec. II. Below we con- 
sider self-similar substitutions in anticipation that they provide a valid leading description of thermalization in the class of models we consider.

Let $n_{0}(k)$ be a distribution function at some late moment of time $t_{0}$, when the regime of self-similarity has been already established. The subsequent evolution can be described as rescaling of momenta accompanied by a suitable change of the overall normalization

$$
n(k, \tau)=A^{\gamma} n_{0}(k A),
$$

where we have defined $\tau \equiv t / t_{0}, \gamma$ is some constant and $A$ $=A(\tau)$ is some time dependent function satisfying $A(1)$ $=1$. Both, $A(\tau)$ and $\gamma$, are determined by the solution of the kinetic equation (6).

In some cases the collision integral may contain an additional explicit time dependence which can be isolated as an overall factor $B(\tau)$. This factor may be induced by timedependent classical backgrounds like the scale factor of the expanding universe or the zero mode of the inflaton field. It is convenient to rescale the collision integral by some typical rate $\Gamma, I \equiv B \Gamma \widetilde{I}$, such that $B$ and $\widetilde{I}$ are dimensionless. We use $B(1)=1$ as normalization.

When Eq. (11) holds, the factor $A^{\gamma}$ of each distribution function, Eq. (29), can simply be taken out of $F$ and out of the collision integral, which becomes a functional of $n_{0}$. After that we can use Eq. (23) with $\xi=A$ which gives

$$
I(k, \tau)=A^{\gamma(m-1)-\mu} B \Gamma \widetilde{I}_{k A}\left[n_{0}\right],
$$

On the other hand, the left-hand side of the kinetic equation (6) can be written as

$$
\dot{n}(k, \tau)=A^{\gamma-1} \dot{A}\left(\gamma n_{0}+\zeta \frac{d n_{0}}{d \zeta}\right),
$$

where we have defined $\zeta \equiv k A$. Using $\Gamma$ as a separation constant, the kinetic equation can be split into two: one for the shape of the distribution function,

$$
\gamma n_{0}+\zeta \frac{d n_{0}}{d \zeta}=-\widetilde{I}(\zeta)
$$

and one for the dynamical evolution

$$
A^{\mu-\gamma(m-2)-1} \frac{d A}{d \tau}=-\Gamma t_{0} B
$$

We will not be concerned with Eq. (32) here and simply assume that it has some nontrivial solution. The general solution of Eq. (33) is of the form

$$
A=\Theta^{-p},
$$

where

$$
\Theta \equiv \frac{\Gamma t_{0}}{p} \int_{1}^{\tau} B\left(\tau^{\prime}\right) d \tau^{\prime}+1
$$

and

$$
p \equiv \frac{1}{\gamma(m-2)-\mu} .
$$

We fix scales using the condition $\Gamma t_{0}=p$. For a timeindependent background $B$, i.e. $B \equiv 1$, it than follows, that $\Theta=\tau$ and Eq. (34) simplifies to

$$
A=\tau^{-p}
$$

We will discuss this case first.

\section{Self-similar evolution in time-independent background}

Substituting Eq. (37) in Eq. (29) we obtain

$$
n(k, \tau)=\tau^{-\gamma p} n_{0}\left(k \tau^{-p}\right),
$$

In applications of turbulence theory to thermalization, this solution is most important. Let $k_{c}$ be the initial value of some characteristic momentum scale, e.g. the scale where most of the energy carried out by a self-similar distribution is concentrated. According to Eq. (38), with time this scale evolves as

$$
k_{c}(\tau)=k_{c}(1) \tau^{p}
$$

The exponent $p$ determines the speed with which the distribution function moves over momentum space and therefore defines e.g. the time scale of thermalization. This is a reason why we will be interested mainly in the value of the exponent $p$, Eq. (36). In applications to thermalization after preheating the energy is concentrated at low momenta initially and should propagate to high momenta. This means that solution Eq. (38) is physically relevant for $p>0$.

The exponent $\gamma$, which enters Eq. (36) can be fixed by specifying appropriate boundary conditions, which are specified below.

a. Isolated systems. If the wave energy is strictly conserved it follows that

$$
\begin{aligned}
\text { const } & =\int d^{d} k \omega_{k} n(k, \tau) \\
& =A^{\gamma-(d+\alpha)} \int d^{d} \zeta \omega_{\zeta} n_{0}(\zeta) .
\end{aligned}
$$

This gives

$$
\gamma=d+\alpha
$$

Similarly, for the evolution with particle number conservation one obtains $\gamma=d$. Here we would like to stress the following subtlety. Clearly, a simple self-similar substitution Eq. (29) cannot account for energy and particle number conservation simultaneously, while both quantities are conserved in a number of systems. If this is the case, one should choose the integral which gives dominant restriction of $n_{k}$, i.e. the energy for energy cascade (thermalization) and particle number for the inverse cascade (Bose condensation). For the problem of thermalization of ultrarelativistic particles this gives 


$$
p_{i}=\frac{1}{(d+1)(m-2)-\mu} \quad \begin{gathered}
\text { relativistic } \\
\text { energy cascade }
\end{gathered}
$$

However, describing thermalization in the nonrelativistic limit, $\omega_{k}=m+k^{2} / 2 m$ with $k^{2} / m^{2} \ll 1$, we can neglect the kinetic energy with respect to the rest mass in the normalization condition (40), i.e. we should use $\gamma=d$, as in the case of particle conservation

$$
p_{i}=\frac{1}{d(m-2)-\mu} \quad \begin{array}{r}
\text { nonrelativistic } \\
\text { energy cascade. }
\end{array}
$$

b. Driven turbulence. In our lattice integrations we have found that particle distributions as functions of $k$ follow a power law in the wake of a propagating energy front, $n_{k}(\tau)=(b(\tau) / k)^{s}$, with exponent $s$ being in agreement with the theoretical predictions for stationary turbulence. Such behavior is expected [86] for the regime of driven turbulence in the presence of a stationary source [and then $b(\tau)=$ const]. However, for the case of free turbulence we are not aware of any predictions. Here we consider consequences of such a behavior assuming general $b(\tau)$ (the case of constant $b$ being a particular case).

Considering distribution the function in the region of low momenta, $n_{k}(\tau)=(b / k)^{s}=A^{\gamma} n_{0}(k A)$ we find

$$
b \propto A^{\gamma / s-1}=\tau^{(1-\gamma / s) p},
$$

i.e. the transport of energy through the inertial range is stationary if

$$
\gamma=s
$$

This generalizes the concept of stationary turbulence to a system without sink. (Notice that this requires a stationary source in the infrared.) In this regime the total energy in particles has to grow linearly with time. Considering the right-hand side of relation (40) with $\gamma=s$ we find $\tau$ $=A^{\gamma-(d+\alpha)}=\tau^{p(d+\alpha-s)}$, or

$$
p_{t}=1 /(d+\alpha-s)
$$

where we denote the exponent $p$ for the case of a stationary transport as $p_{t}$ to distinguish it from the exponent which corresponds to an isolated system, $p_{i}$. Substituting explicitly the exponent $s$ of the spectra of stationary turbulence, Eq. (26), we find

$$
p_{t}=\frac{(m-1)}{(d+\alpha)(m-2)-\mu}=(m-1) p_{i} .
$$

The latter relation could have been also found using Eq. (26) and Eqs. (36) with $\gamma=s$.

c. Nonstationary source. Let us consider the somewhat more general situation and assume that the energy inputted into (or taken out from) the system of particles changes with time as $E(\tau)=E_{0} \tau^{r}$. Clearly, the isolated system corresponds to $r=0$, while a stationary source corresponds to $r$ $=1$. We will now have $\gamma=(d+\alpha)-r / p$ and

$$
p=\frac{1+r(m-2)}{(d+\alpha)(m-2)-\mu} .
$$

\section{Time-dependent background}

We now consider a time-dependent $B$ in Eqs. (34), (35). As an illustration we choose $B(\tau)=\tau^{-\kappa}$, which gives

$$
\Theta=\frac{1}{(1-\kappa)}\left(\tau^{1-\kappa}-1\right)+1
$$

Note, and this is important for the interpretation of our numerical results, that the linear approximation for small times, $\tau \sim 1$, gives $\Theta \simeq \tau$, which brings us back to the situation considered in the previous subsection.

The late time behavior, $\tau \gg 1$, depends on the sign of 1 $-\kappa>0$. If $1-\kappa>0$, the distribution propagates to the ultraviolet without bound, $A(\tau) \propto \tau^{-(1-\kappa) p}$ and $k_{c}(\tau) \propto \tau^{(1-\kappa) p}$. In other words, at late times $A \sim \tau^{-\tilde{p}}$ with

$$
\tilde{p}=(1-\kappa) p
$$

for any boundary conditions discussed above in Secs. III C 1 a-III C 1 c.

However, $A(\tau)$ approaches a finite limit at $\tau \rightarrow \infty$ if 1 $-\kappa<0$,

$$
A(\tau=\infty)=\left[1+\frac{1}{\kappa-1}\right]^{-p}
$$

The propagation of particle distribution functions towards the ultraviolet is limited. This has important consequences for the thermalization of massive particles in the expanding Universe, as we shall discuss in more detail below.

Expressions Eqs. (42), (47) and (50) are the main results of this section. They determine the speed of propagation of the particle distribution in momentum space for a specific models.

\section{STATIONARY STATES AND SELF-SIMILAR EVOLUTION IN SPECIFIC MODELS}

Here we apply the general results of the previous section to a number of particular models of interest. First of all we have to determine the scaling exponent $\mu$ of $d \Omega$ [see Eq. (21)]. The scaling of $\omega$ is different in relativistic and nonrelativistic regimes. This is accounted for differently in the argument of the energy conservation $\delta$ function [where in the nonrelativistic regime $\omega(k)$ is replaced by $\left.k^{2} / 2 m\right]$ and in the $1 / \omega$ factors of relativistic integration measure (where $\omega$ is replaced by $m$ ). To make the discussion of relativistic and nonrelativistic cases uniform, we move $\omega$ out from the relativistic integration measure and define the function $U\left(k, q_{i}\right)$,

$$
U\left(k, q_{i}\right) \equiv \frac{(2 \pi)^{d+1}\left|M_{k}\right|^{2}}{2 \omega_{k} \prod_{i=1}^{m-1} 2 \omega_{q_{i}}}
$$


In what follows we will assume that in a dynamically interesting range of wave numbers $U$ follows a scaling law

$$
U\left(\xi k, \xi q_{i}\right)=\xi^{\beta} U\left(k, q_{i}\right) .
$$

With this definition

$$
d \Omega\left(k, q_{i}\right)=U\left(k, q_{i}\right) \delta^{d}\left(k_{\mu}, q_{i \mu}\right) \prod_{i=1}^{m-1} \frac{d^{d} q_{i}}{(2 \pi)^{d}},
$$

and we find

$$
\mu=d(m-2)-\alpha+\beta .
$$

We calculate the exponents $\mu, s$, and $p$ for two classes of models. The first one is characterized by $k$-independent matrix elements, the second one has no dimensionful parameters. The scalar field models which we integrated on the lattice belong to the first class. In the absence of a zero mode in the relativistic limit in $(3+1)$ dimensions they belong to the second class as well.

\section{A. Theory with $k$-independent matrix elements}

For models with k-independent matrix elements the scaling of $U$ is determined by the $\omega$ 's, and we have $\beta=-m$ in the relativistic regime and $\beta=0$ in the nonrelativistic case. Equation (55) gives

$$
\begin{aligned}
& \mu=d(m-2)-1-m \quad \text { (relativistic), } \\
& \mu=d(m-2)-2 \quad \text { (nonrelativistic). }
\end{aligned}
$$

Substituting these expressions into Eqs. (42), (43) we find that in this class of models the exponents $p$ do not depend on the number of dimensions. In particular, for the energy cascade in an isolated system we have

$$
\begin{aligned}
& p_{i}=1 /(2 m-1) \quad \text { (relativistic), } \\
& p_{i}=1 / 2 \quad \text { (nonrelativistic). }
\end{aligned}
$$

For $m=3$ and $m=4$ Eq. (58) gives $p=1 / 5$ and $p=1 / 7$ respectively.

Substituting Eqs. (56), (57) into Eq. (26) we find the exponent $s$,

$$
\begin{aligned}
& s=d-\frac{m}{m-1} \quad \text { (relativistic), } \\
& s=d \quad \text { (nonrelativistic). }
\end{aligned}
$$

In the nonrelativistic regime both exponents, $p_{i}$ and $s$ do not depend on $m$.

\section{Three-particle interactions, relativistic regime}

Three-particle processes appear in the $\lambda \phi^{4}$ model when interactions with the zero mode are important, see Appendix $\mathrm{B}$ and Sec. IV C 1.

According to Eq. (58) for $m=3$ the front of the energy cascade propagates with

$$
p_{i}=\frac{1}{5}
$$

regardless of the number of spatial dimensions, $d$. For the exponent $s$ of particle distributions in the inertial range in $d=3$ we find

$$
s=\frac{3}{2} .
$$

Both exponents coincide with what is observed in our numerical experiments. Note that the exponent $s$ is expected to appear in the case of driven turbulence. In the case of free turbulence the wake of the propagating turbulent front does not even have to be a power law. Nevertheless, we do observe a power law with the exponent $s=3 / 2$ to a very good accuracy. This might be not a chance coincidence. However, in $d<3$ the theory predicts $s<1$, the spectrum falling off with $k$ more slowly compared to thermal equilibrium, and one can get a different shape of particle distributions in $d$ $<3$ [but we still expect the exponent $p$ to be given by Eq. (58)].

\section{B. Relativistic theory with dimensionless couplings}

The $\lambda \phi^{4}$ model in $d=3$ which we have simulated on the lattice belongs to the class of models considered in this paragraph. In $d=2$ dimensionless couplings appear in the $\lambda \phi^{6}$ model. Dimensionless couplings are generic and this case is not restricted to scalar field models, therefore we consider it separately.

If the collision integral does not contain any dimensionfull parameters, it has to scale with $\mu=1$ and we find for the exponent $p_{i}$ of energy conserving propagation in an isolated system, Eq. (42)

$$
p_{i}=\frac{1}{(d+1)(m-2)-1} .
$$

For the physical case of $d=3$ and for a 4-particle processes (which should dominate at late times in the models we have considered numerically, see below) we obtain

$$
p_{i}=\frac{1}{7}
$$

Note that for $d=2$ and $m=6$ we have $p_{i}=1 / 11$, in agreement with Eq. (58). For the exponent $s$ of particle distribution functions in the energy cascade we find, see Eqs. (26)

$$
s=\frac{d+2}{m-1}=\frac{5}{3} .
$$

\section{Explicit time dependence in the collision integral}

The self-similar evolution is modified when an explicit time dependence is present. Below we consider two specific models with explicit time dependence in the collision integrals which appear in the problem of reheating. The first one is directly related to the relativistic scalar model we have 
simulated on the lattice and time dependence enters via the coupling to the zero mode. The second describes thermalization of nonrelativistic particles and the time dependence is induced by the expansion of the Universe.

\section{Nonzero classical field}

Typically, oscillations of the inflaton zero mode do not decay completely during the initial stage of parametric resonance. Moreover, if the resonance parameter is large, parametric decay stops early, when only a small part of the initial inflaton energy has been transferred to particles [9]. The remaining oscillating zero mode serves as a source in our turbulent problem. This source acts via two different channels. The first one can be described as a direct decay into the resonance band(s). The other channel is $\mathrm{m}$-particle scattering when one or more particles have zero momentum. These particles belong to the zero mode (which is a Bose condensate). While the zero mode and excitations with $\boldsymbol{k} \neq 0$ can be viewed as the same particles but with different momentum, the formal description is different. The presence of the zero mode $\phi_{0}$ leads to new specific terms in the collision integral with reduced number of particles participating in the interaction process and different (and time-dependent) couplings.

The simplest example is 2 by 2 scattering in the $\lambda \phi^{4}$ model when one of the incoming or outcoming particles belongs to the condensate. These scattering processes can be modeled as an effective 3-particle interaction. The corresponding 3-particle collision integral can be obtained from the 4-particle one with the substitution

$$
\frac{n_{p}}{\omega_{p}} \rightarrow \frac{n_{p}}{\omega_{p}}+(2 \pi)^{3} \delta^{(3)}(\mathbf{p}) \bar{\phi}_{0}^{2} .
$$

This gives an explicit time dependence in front of the collision integral, $B=\phi_{0}^{2}(\tau) / \phi_{0}^{2}(1)$, and reduces the number of integrations by one, $m=3$. Alternatively, the 3-particle collision integral in the background of a zero mode can be derived from first principles, see Appendix B.

The turbulent exponents for the 3-particle scattering without explicit time dependence [i.e. $\phi_{0}^{2}(\tau)=1$ ], are given by Eqs. (62) and (63). Both agree with what is observed in our numerical experiments, see Sec. II. We show in Sec. VI that the collision integral in our lattice problem is dominated by 3-particle interactions. Therefore, Eq. (63) for the exponent $s$ seems to be indeed applicable for the system considered numerically. The question of applicability of Eq. (62) for the exponent $p$ deserves special consideration because the amplitude of the zero mode changes with time.

During the initial stage, when the total energy in particles is small compared to the energy stored in the zero mode, we can consider the amplitude of oscillations to be constant and the source of turbulence to be stationary. However, distribution functions should then evolve with $p_{t}=2 p_{i}$, see Eq. (47). At late times on the other hand we cannot neglect the decay of the zero mode. Numerical integrations show that the amplitude of the zero mode decreases as a power law, $\phi_{0}^{2}(\tau)$ $\propto \tau^{-\kappa}$. At late times this gives $p \rightarrow(1-\kappa) p$, see Eq. (50). Numerically $\kappa=2 / 3$, however, the conclusion that $p=1 / 15$ would be incorrect. First, for completely decayed zero mode the 4-particle collision would dominate, leading to $p=1 / 7$. Therefore, in our problem we should expect $p \geqslant 1 / 7$ at all times. Second, the condition $\tau \gg 1$ is not fulfilled during our integration time. Indeed, we observed self-similarity for $3600<\eta<10000$, see Fig. 3, which corresponds to $\tau<3$. For $\tau \approx 1$ the solution of Eq. (34), (49) for $A(\tau)$ coincides with $A=\tau^{-p}$, while at $\tau=3$ it deviates by not more than $5 \%$. Therefore, in this time interval $A(\tau) \approx \tau^{-1 / 5}$. Similarly, the quantity $A^{\gamma}$ with $\gamma=4$ for $1<\tau<3$ (energy conservation) is close numerically to $\tau^{-q}$, where $q \simeq 3 / 5$. Hence the indices of self-similar evolution obtained in Sec. II are explained by free turbulence driven by three-particle interactions in the background of zero mode.

\section{Nonrelativistic regime in expanding universe}

Let us consider now non-relativistic particles in an expanding universe with physical dimension $d=3$. We will be working in the conformal reference frame, Eq. (1). In these coordinates the expansion of the universe is simply accounted for by multiplying all bare mass parameters, $M$, by the scale factor. This is true both for the original field equations and for the kinetic equations (which are derived from the former). Factors of $\omega$ in the measure Eq. (52) should be replaced by $M a(\eta)$. Therefore, in the nonrelativistic regime the collision integral in the expanding universe can be obtained by multiplying it by the scale factor in some negative power.

In conformal reference frame the solution of the Friedmann equations for the scale factor as a function of $\tau$ $\equiv \eta / \eta_{0}$ can be written as

$$
a^{b}=b H_{0} \eta_{0}(\tau-1)+1,
$$

where $H_{0}$ is the value of the Hubble parameter at time $\eta_{0}$. For the radiation dominated expansion $b=1$, while $b=1 / 2$ for the matter dominated expansion. Hence, the function $B(\tau)$ takes the form

$$
B(\tau)=\left[b H_{0} \eta_{0}(\tau-1)+1\right]^{-\kappa} .
$$

where $\kappa=3 / b$ for the 4 -particle process in $\lambda \phi^{4}$ theory, i.e. $\kappa=3$ and $\kappa=6$ for radiation and matter dominated expansion respectively. This gives

$$
\int_{1}^{\tau} B\left(\tau^{\prime}\right) d \tau^{\prime}=\frac{1-\left[b H_{0} \eta_{0}(\tau-1)+1\right]^{1-\kappa}}{b(\kappa-1) H_{0} \eta_{0}} .
$$

We see that in the limit $\tau \rightarrow \infty$

$$
A(\tau=\infty)=\left[1+\frac{1}{b(\kappa-1) H_{0} \eta_{0}}\right]^{-p}
$$

where $p$ is given by Eq. (43). The particle distributions cannot propagate to high momenta and are frozen out at

$$
k_{c}(\tau=\infty)=\frac{k_{c}(1)}{A(\tau=\infty)}=\frac{k_{c}(1)}{\left[b(\kappa-1) H_{0} \eta_{0}\right]^{p}} .
$$


In the traditional discussion of thermalization of particles in the expanding Universe, see e.g. Ref. [2], the expansion rate, $H_{0}$, is compared to the to the rate of interactions, which in our case can be identified with $\eta_{0}$ [see the normalization factor in Eq. (34)]. It is concluded that particles can not thermalize if $H_{0} \eta_{0}>1$ while they can reach thermal equilibrium when $H_{0} \eta_{0}<1$. Equation (72) tells us that thermalization is indeed impossible for $H_{0} \eta_{0}>1$ since the distributions do not move towards high momenta in this case. However, it is not guaranteed that the equilibrium is reached even if $H_{0} \eta_{0} \ll 1$. The system may thermalize only if $k_{c}(\tau=\infty)$ is not smaller than the typical values of momenta in eventual thermal equilibrium.

\section{TWO INTERACTING SCALAR FIELDS: NUMERICAL RESULTS}

In this section we present the results of lattice calculations of reheating in the model of two interacting fields. As in the one field model presented in Sec. II, we again consider the massless case, for which the use of conformal transformation allows mapping of the dynamics in expanding Friedmann universe into the case of Minkowski space-time. This permits a long integration time on a fixed lattice.

\section{A. Model}

At the end of inflation the universe is very close to a spatially flat Friedmann model. It is convenient to work in conformal coordinates where the metric takes the form $d s^{2}$ $=a(\eta)^{2}\left(d \eta^{2}-d x^{2}\right)$. We consider two scalar fields $\Phi$ and $X$ whose dynamics are determined by the action $\mathcal{S}$ $=\int d t d^{3} x \sqrt{-g} \mathcal{L}(\Phi, X)$ with Lagrangian density

$$
\mathcal{L}=\frac{1}{2} g^{\mu \nu} \partial_{\mu} \Phi \partial_{\nu} \Phi+\frac{1}{2} g^{\mu \nu} \partial_{\mu} X \partial_{\nu} X-V(\Phi, X)
$$

and potential

$$
V(\Phi, X)=\frac{\lambda_{\Phi}}{4} \Phi^{4}+\frac{\lambda_{\Phi X}}{2} \Phi^{2} X^{2}+\frac{\lambda_{X}}{4} X^{4} .
$$

We identify the field $\Phi$ with the inflaton. Therefore $\lambda_{\Phi}$ $\simeq 10^{-13}[1-3]$. Inflation ends at time $\eta_{0}$ when $\left\langle\Phi\left(\eta_{0}\right)\right\rangle$ $\simeq 0.35 \mathrm{M}$ Pl.

We use the following set of coordinate and field rescalings which bring the system into a dimensionless form suitable for numerical integration:

$$
\begin{aligned}
& \left.\begin{array}{r}
d x_{0} \\
d \mathbf{x}_{i}
\end{array}\right\} \rightarrow\left\{\begin{aligned}
d \eta & \equiv \frac{d x_{0}}{a} \lambda_{\Phi}^{1 / 2} \Lambda \\
d \mathbf{y}_{i} & \equiv d \mathbf{x}_{i} \lambda_{\Phi}^{1 / 2} \Lambda,
\end{aligned}\right. \\
& \left.\begin{array}{c}
\Phi \\
X
\end{array}\right\} \rightarrow \begin{cases}\varphi & \equiv \Phi \Lambda^{-1} a(\eta) \\
\chi & \equiv X \Lambda^{-1} a(\eta) .\end{cases}
\end{aligned}
$$

Re-scaling of the fields with $a(\eta)$ in Eq. (76) rotates the scale factor away and maps the model into a scalar field theory in Minkowski space-time. The classical equation of motion have two independent parameters

$$
g \equiv \lambda_{\Phi X} / \lambda_{\Phi}, \quad h \equiv \lambda_{X} / \lambda_{\Phi}
$$

and simplify to

$$
\begin{aligned}
& \square \varphi+\varphi^{3}+g \chi^{2} \varphi=0, \\
& \square \chi+h \chi^{3}+g \varphi^{2} \chi=0 .
\end{aligned}
$$

We choose $\Lambda=\left\langle\Phi\left(\eta_{0}\right)\right\rangle$, so that the initial condition for the inflaton zero mode reads $\left\langle\varphi\left(\eta_{0}\right)\right\rangle=1$. Equations (78) and (79), however, are independent on the particular choice of $\Lambda$. At $\eta=\eta_{0}$ all correlation functions of $\Phi$ and $X$ on subhorizon scales characterize a vacuum of fluctuations around the inflaton mean value.

\section{B. Results of numerical integration}

We have studied the two-field model using the following set of coupling constants: $\lambda_{\Phi}=10^{-13}, g=30$, and $h$ was varied in the range $0.1 g \leqslant h \leqslant 10^{4} \mathrm{~g}$. We will see below that different values of $h$ lead to different duration and different relative importance of the specific dynamical regimes, as it was already argued for in Sec. II B. These are: the regime of parametric resonance, the regime of stationary (or driven) turbulence and the regime of free turbulence. These issues will be addressed later in this section, which we start with the discussion of particle spectra.

\section{Spectra}

The particle spectra in the two field model at late times are very similar to what we have observed in the one field model and have the same turbulent exponents. Namely, in the inertial range $n_{k}$ is a power low with the exponent $s$ $=3 / 2$, for both fields $\chi$ and $\phi$, see Fig. 4. And both fields evolve in a self-similar way with $p=1 / 5$ at sufficiently late times, when the energy in particles became comparable to the energy in the zero mode, see Fig. 5. Both exponents, $s$ and $p$, correspond to turbulence supported by 3-particle interactions.

There are some differences however. For the considered range of parameters, the coupling of the excitations to the medium is rather strong, which induces large effective particle masses, see Appendix A 3. Therefore particles are nonrelativistic already in the part of the inertial range. Namely, $M_{\chi} \simeq 5.5 \bar{\phi}$ and $M_{\phi} \simeq 1.7 \bar{\phi}$. This manifests itself as $\sim k^{-3}$-power-law behavior, which is again consistent with domination of 3-particle interactions, see Eq. (59). This can be expressed as a single power law if particle distributions are plotted as functions of relativistic kinetic energy,

$$
\epsilon_{k} \equiv \omega_{k}-M
$$

where $M$ is the effective particle mass. Indeed, in the relativistic region we have $n_{k} \propto k^{3 / 2} \propto \epsilon_{k}^{3 / 2}$, while in the nonrelativistic region we obtain $n_{k} \propto k^{3} \propto \epsilon_{k}^{3 / 2}$. For this reason, the particle distributions were plotted in Fig. 4 as functions of $\epsilon_{k}$. The particle distributions for the $\chi$ field appear in this variable as featureless single power law. This can be easily understood. First, the energy transport for 3-particle interactions in the presence of zero mode corresponds to the 

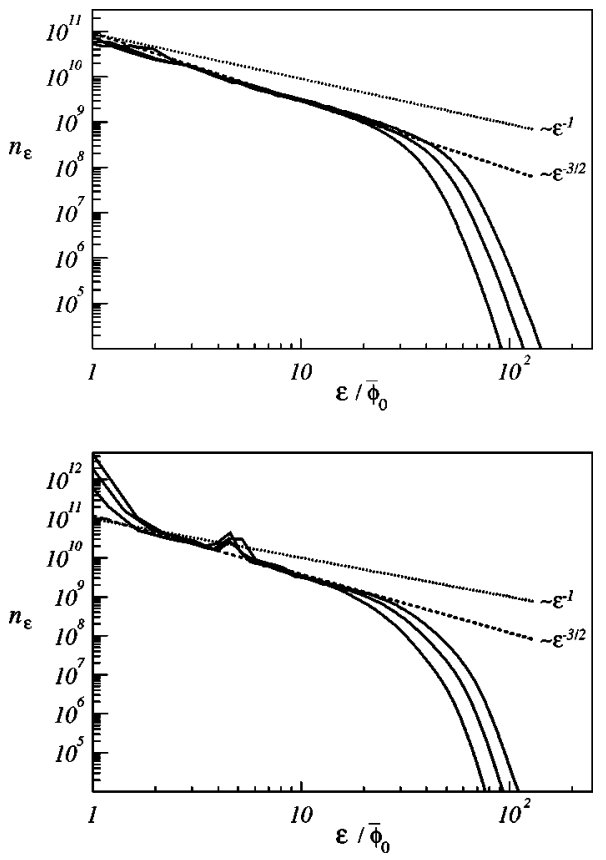

FIG. 4. Particle distributions in the self-similar regime for $h$ $=10 \mathrm{~g}$ as functions of the corresponding wave kinetic energies rescaled by the current zero-mode amplitude $\bar{\phi}_{0}$. Upper and lower panels correspond to $\chi$ and $\phi$ fields respectively. In both cases from left to right the plots are taken at $\eta=1000,1500,2000$.

transport of kinetic energy, as energy conservation law in elementary scattering process, which involves the frequency of zero-mode oscillations, $\omega_{0} \approx M$, tells us, see Appendix B. Second, the collision integral, Eq. (B38), being substituted into expression for the energy flux, Eq. (15), will have appropriate universal scaling behavior in terms of kinetic energy, $\epsilon_{k}$, but not in terms of $k$. Therefore, the kinetic energy is indeed the appropriate variable for the case of 3-particle interactions in the presence of zero mode.

For $h>g$ the spectra look stationary in the inertial range after rescaling $\epsilon$ by the current zero-mode amplitude $\bar{\phi}_{0}$ $\sim \eta^{-1 / 3}$. This is similar to the one field case (see Fig. 4). However, for $h \leqslant g$ we found $\bar{\phi}_{0} \sim \eta^{-2 / 3}$, but the spectra still appear stationary after rescaling by $\eta^{-1 / 3}$. This can be understood in the light of Eq. (44): $b(\tau)=\tau^{-1 / 3}$ is consistent with the choice $\gamma=4, s=3 / 2$ and $p=1 / 5$. Hence, the decreasing amplitude of distribution functions in the region of low $k$ simply reflect the energy conservation in the system.

\section{Stationary and free turbulence regimes}

Let us demonstrate now that the regime of stationary turbulence does occur in the two field model. This regime is expected to appear in the case of large values of dimensionless parameters, $g \gg 1, h \gg 1$, when parametric resonance stops early, while the total energy is still stored in the zero mode.

We found that in the relevant range of parameters the description in terms of particles, which we were using so far, deteriorates. The reason is that in this language at large couplings there is no unique way to split the total energy density
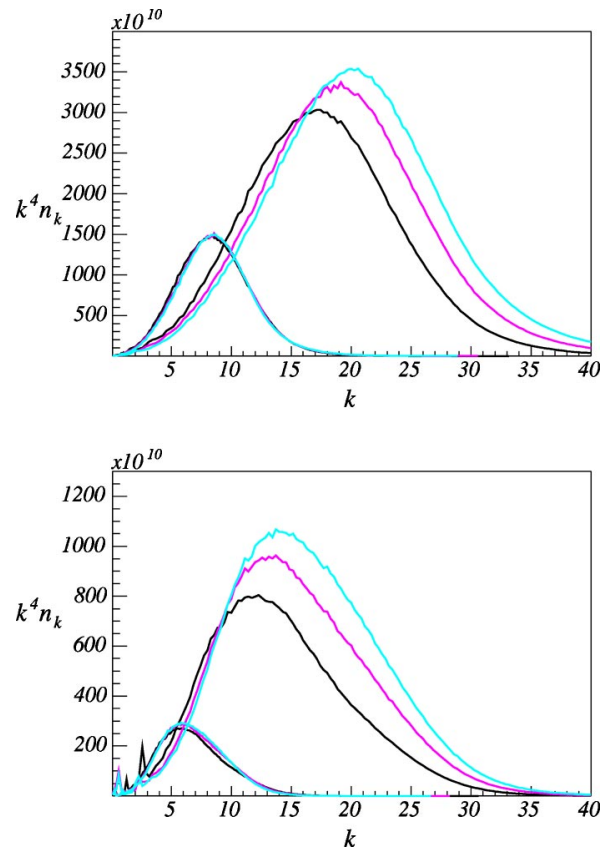

FIG. 5. Spectral energy distributions for $\chi$ (upper panel) and $\phi$ (lower panel) in the model with $h=10 \mathrm{~g}$. In each panel we plot the wave energy per decade found in lattice integrations at three moments of time, $\eta=1000,1500$ and 2000. In the lower-left corner of each panel are the same graphs transformed according to the relation inverse to Eq. (4).

of the system into contributions coming from zero mode and fluctuation field.

To deal with this problem we have quantified the energy transfer in the following way. The quantity $\rho_{0} \equiv \frac{1}{2} \dot{\phi}_{0}\left(\eta_{z}\right)^{2}$ gives a good measure of the total energy density stored in zero-mode oscillations, if it is measured at those moments of time, $\eta_{z}$, when the mean field crosses zero, $\phi_{0}\left(\eta_{z}\right)=0$. In this way we can get rid of the ambiguity in accounting interaction energy between zero mode and fluctuations. Similarly, we measure the energy density in the fluctuation field as $\rho_{\chi}$ $\equiv\left\langle\dot{\chi}^{2}\right\rangle_{t}$ and $\rho_{\phi} \equiv\left\langle\dot{\phi}^{2}\right\rangle_{t}$ for the $\chi$ and $\phi$ fields respectively. Here $\langle\ldots\rangle_{t}$ means lattice and time averaging. We verified numerically that the sum of these quantities conserves with time and equals to the initial energy density. This is not true, however, when we measure the energy density in particles as $\omega_{k} n_{k}$. Both measures of particle energy converge at late times when the interaction energy becomes unimportant.

This "kinetic" measure of the total energy density stored in particles as a function of time is shown in Fig. 6. We compare models with two different values of $h$. Three different regimes are clearly seen in both cases.

(i) Parametric resonance: The energy density $\rho_{\chi}$ grows exponentially. This regime continues until re-scattering becomes important. The larger $h$ is, the earlier resonance terminates.

(ii) Stationary turbulence: At later time the energy density in $\chi$ particles grows linearly in time, which according to Eq. (5) is a sign of stationary turbulence. During this period the energy density still stored in the zero mode dominates the total energy balance. 


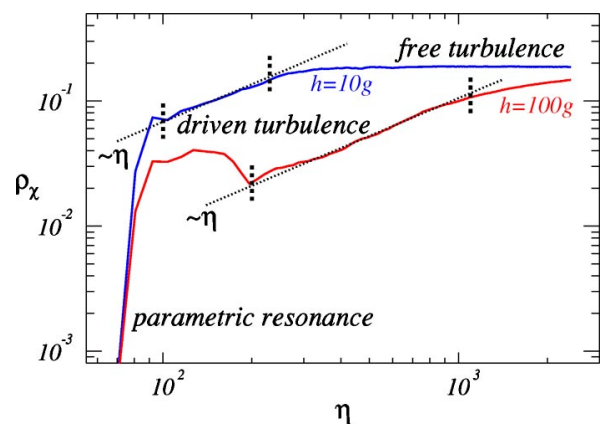

FIG. 6. Different regimes of the evolution of the $\chi$ field for two values of self-coupling, $h=10 g$ and $h=100 g$. The dashed lines correspond to a linear growth of energy in the $\chi$ field with time, $\rho_{\chi} \propto \eta$.

(iii) Free turbulence: At some point the energy density in the zero mode drops below the energy density already stored in particles. Stationary turbulence cannot be sustained anymore and the regime of free turbulence, with conserved energy in particles, follows. We may expect self-similar evolution of particle distribution functions, which at late times are good quantities.

In the model with larger self-coupling the parametric resonance stops earlier and only a negligible part of the inflaton energy is transferred to particles during the resonance stage, see Fig 6. In this parameter range the transfer of energy from the inflaton into $\chi$ field is dominated by a stationary turbulence. In the Sec. VII B we show that if all coupling constants are of order of the inflaton self-coupling, the thermalization is a very long process and the Universe reheats to unacceptably low temperature, $T \sim 100 \mathrm{eV}$. Therefore, some couplings in the sector of physical fields (e.g. self-couplings, or couplings to the inflaton) in a realistic model have to exceed significantly the scale of the inflaton self-coupling. With larger couplings the thermalization proceeds faster. This is confirmed in our lattice integration, see Fig. 7. At earlier times the model with larger self-coupling contains less energy in $\chi$ particles, cf. curves at $\eta=500$. However, at later times this model takes over and the energy containing region moves faster towards ultraviolet in the model with larger self-coupling.

With even larger self-coupling of the $\chi$ field, or its coupling to the inflaton, the period of stationary turbulence

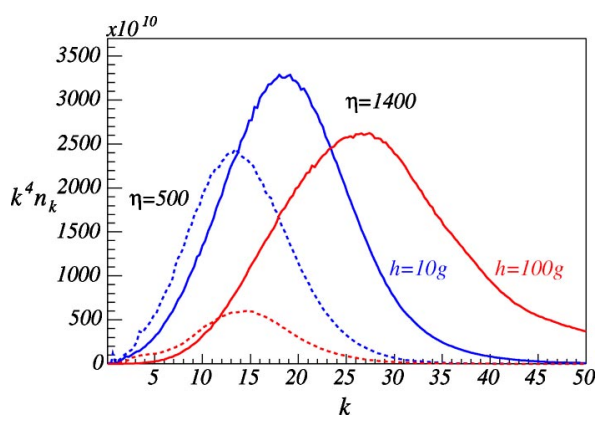

FIG. 7. Spectral energy distributions at two moments of time, $\eta=500$ (dotted lines) and $\eta=1400$ (solid lines). We compare two models with different self-coupling, $h=10 g$ and $h=100 g$. should become even more pronounced. In light of these findings, we can also understand the results of earlier papers $[9,10]$. E.g., in figures presented in Ref. [10], we see clear signs of driven turbulence, which was not identified as such until now. In particular, in Ref. [10] it was found that the energy in $\chi$ fluctuations grows with time as $\rho_{\chi} \propto t^{0.95}$. (Small deviation from $\propto t$ law can be due to the fact that the energy in zero mode decreases somewhat and the source deviates from stationarity.) This regime persists until the final integration time, when distribution functions reach the boundary of the integration box, and even then the system is far from free turbulence regime.

We conclude that in the models with an acceptable reheating temperature, the parametric resonance stops only when a negligible fraction of the inflaton energy has decayed. Therefore, in realistic models of the type considered in the present paper, the major mechanism of energy transfer from the inflaton into particles is stationary turbulence.

\section{IS THE KINETIC APPROACH APPLICABLE?}

In this section we confront the results of our lattice integration with the predictions of kinetic theory and address the validity of the kinetic description at the thermalization stage during our integration time interval.

The particle distributions in the inertial range, $n(k)$ $\sim k^{-s}$ with $s \approx 3 / 2$, which we observe in the lattice simulations, can be understood as corresponding to the scale invariant energy flux for 3-particle interactions, see Eq. (63). The observed exponent $p=1 / 5$ of the self-similar scaling of free turbulence, can also be in accord with 3-particle interactions, see Eq. (62). However, in our case bare 3-particle couplings are absent and appear effectively in interactions with zero mode. Therefore, the 3-particle collision integral is multiplied by the amplitude of zero mode squared. Since the amplitude of the zero mode oscillations decays, one can expect $p=1 / 5$ only during a small time interval, see Sec. III C 2.

Can 4-particle interactions be responsible for the observed scalings? For 4-particle interactions $p_{i}=1 / 7$, see Eq. (65), which is not that far away from the lattice results, especially if one takes into account energy influx from the zero mode. However, for particle distributions in the inertial range one should expect $s=5 / 3$, which is not in a good agreement with the observed value of $s=3 / 2$. Further, in view of Eq. (67) one should expect the dominance of 4-particle scattering during the time interval when the variances of fluctuations are larger than $\phi_{0}^{2}$. This is not the case during the time interval encompassed by the lattice simulations, see Fig. 1.

The outlined difficulties may give an indication that the weak turbulence description is not applicable in our case. In view of the importance of the issue, we performed a detailed study of collision integrals, anomalous and higher order correlators, as measurements on the lattice, and compared these with predictions and assumptions of kinetic theory.

\section{A. Collision integrals}

To verify the extent of agreement between kinetic theory and lattice calculations, and to find out which processes 


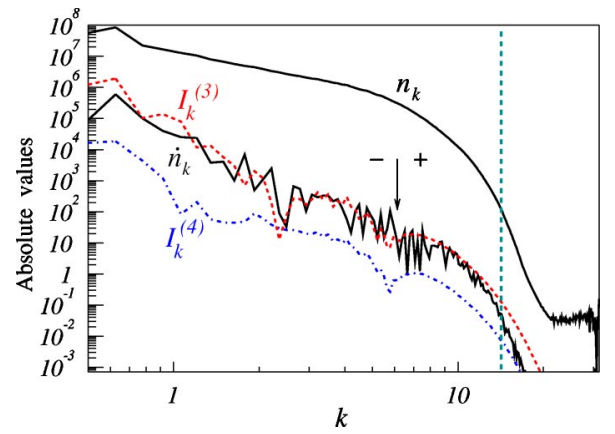

FIG. 8. Absolute values of $\dot{n}(k)$ and of $I_{k}^{(3)}$ and $I_{k}^{(4)}$ collision integrals at $\eta=5000$. To the left of the arrow $\dot{n}(k)$ and collision integrals are negative, while to the right they are positive. Occupation numbers, $n_{k}$, are also shown for comparison. $I_{k}^{(3)}$ agrees with $\dot{n}(k)$ to the left of the vertical dashed line.

dominate the collision integral in our problem, we carry out the following procedure. First, we numerically calculate the collision integrals using standard expressions, Eqs. (7)-(10), and the particle distribution functions $n_{k}(\eta)$ extracted from our lattice calculations. Second, using lattice data we calculate time derivatives of the distribution functions to see if the relation $\dot{n}_{k}=I_{k}[n]$ holds. We limit ourselves to 3 - and 4-particle collisions.

The general relations, Eqs. (7)-(10), for 4- and 3-particle collision integrals can be reduced to two and one dimensional integrations respectively, if the distribution functions are isotropic. Explicit expressions are given in Appendix B, Eqs. (B38) and (B39).

The numerically calculated values of $I_{k}^{(3)}$ and $I_{k}^{(4)}$ collision integrals are shown in Fig. 8 in comparison with $\dot{n}_{k}$. Note that the collision integrals and $\dot{n}_{k}$ take positive and negative values. For clarity we show only absolute values of these functions and indicate schematically the boundary between regions where $\dot{n}_{k}$ is negative and positive. Roughly, in the inertial range $\dot{n}_{k}$ is negative (recall that in this region the particle distributions can be approximated as $n_{k}(\eta)$ $=\left(\bar{\phi}_{0} / k\right)^{s}$ and are decreasing functions of time), while $\dot{n}_{k}$ should be positive at larger $\mathrm{k}$ where the cutoff starts (recall that energy is flowing into this region).

We find that $I_{k}^{(3)}$ gives a reasonable approximation to $\dot{n}_{k}$ practically in all range of $k$ which is dynamically important, which is to the left of the vertical dashed line in Fig. 8. One reason for the disagreement between $\dot{n}(k)$ and $I_{k}^{(3)}$ at larger $k$ could be due to the fact that on the lattice some of the allowed resonant wave interactions of the continuum limit are not present (cf. Ref. [88]). In any case, in the region where $I_{k}^{(3)}$ and $\dot{n}_{k}$ disagree, the occupation numbers are relatively small, $n_{k}<10^{2}$, and this region should not contribute to the dynamics significantly.

The $I_{k}^{(4)}$ collision integral is about an order of magnitude smaller compared with $I_{k}^{(3)}$ and is subdominant in the evolution of $n_{k}$, except on the very tail of the distribution, see Fig. 8. The agreement between $\dot{n}_{k}$ and $I_{k}^{(4)}$ in the region of the tail is not coincidental-we observe it at all $\eta$.

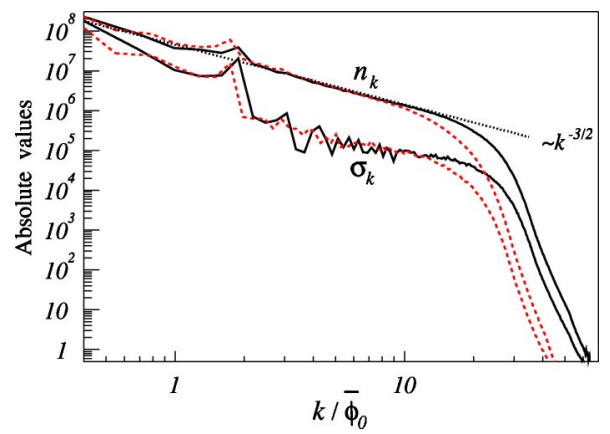

FIG. 9. Occupation numbers and absolute values of $\sigma_{k}$ at $\eta=5000$ (dashed lines) and $\eta=10000$ (solid lines).

\section{B. Anomalous correlators}

Usually, kinetic equations are derived under the assumption $\left\langle a_{k} a_{q}\right\rangle \ll\left\langle a_{k}^{*} a_{q}\right\rangle$. However, this condition not always holds. For example, in the case of particle creation by a time-varying classical background (e.g. in the region of parametric resonance)

$$
\dot{n}_{k}=\frac{\dot{\omega}_{k}}{\omega_{k}} \operatorname{Re}\left(\sigma_{k}\right),
$$

where

$$
\left\langle a_{k} a_{q}\right\rangle \equiv \sigma_{k} \delta(\mathbf{k}+\mathbf{q}),
$$

see Appendix B. In this case the anomalous correlators, $\sigma_{k}$, can not be neglected, since $\sigma_{k} \sim n_{k}$. This holds in general: if coherent processes are important, the correlators Eq. (82) may modify the dynamics of $n_{k}$. If this is the case, they should be included into the kinetic equation. Since $\sigma_{k}$ were neglected in the kinetic equations, Eqs. (7)-(10), it is important to verify if the condition $\left|\sigma_{k}\right| \ll n_{k}$ holds in our simulations.

The correlators $\sigma_{k}$ are shown for several moments of time in Fig. 9. In the inertial range the anomalous correlators are small indeed, $\left|\sigma_{k}\right| / n_{k} \approx 3 \times 10^{-2}$, while this ratio is an order of magnitude larger in the region of the resonance peak $(k$ $\approx 0.5$ at late times), which is expected behavior. The ratio $\left|\sigma_{k}\right| / n_{k}$ is growing also in the region of large $k$, reaching the value of 0.1 at $k=8$ at late times, see Fig. 3. To avoid confusion, note that $k=8$ corresponds to $k / \bar{\phi}_{0} \approx 25$, which is the variable used in Fig. 9. We do not know if the growth of $\left|\sigma_{k}\right| / n_{k}$ at large $k$ is a lattice effect, but we can conclude that the kinetic equations in its simple form, Eqs. (7)-(10), should be applicable in the inertial range.

\section{PHYSICAL APPLICATIONS}

Many different effects may occur during the stage of preheating. Some of these were discussed in the Introduction section. They have a common physical origin: rapid particle creation and large accompanying fluctuations of the classical fields involved. These findings are unaffected by our results, even in the case when only a relatively small fraction of the inflaton energy is transferred to fluctuations during the initial stage of parametric resonance. 
However, in many cases it is necessary to trace the events further in time, e.g. to find out when and how the symmetry which was restored during preheating gets broken later on, or to trace which fraction of baryon or lepton asymmetry survives in the process of thermalization. Finally, one needs to know when thermal equilibrium will be established. This gives e.g. the abundances of particular dark matter particle candidates and other, possibly cosmologically "dangerous" relics like the gravitino.

The explicit time dependence of the particle distribution functions, and the knowledge that the evolution is selfsimilar, $n(k, \tau)=A^{\gamma} n_{0}(k A)=\tau^{-\gamma p} n_{0}\left(k \tau^{-p}\right)$, which we have found in the present paper, may be useful here. Below we discuss some applications, limiting ourselves to field variances and to the problem of thermalization.

\section{A. Field variances}

In some applications, basic observables like field variances may already give the answer to the problem in question. This applies to the problem of symmetry restoration. To illustrate this, let us consider the Higgs field which is coupled to a $\chi$ field. In the vacuum state without condensate the mass squared of the Higgs field would be negative, $-\mu^{2}$, and the corresponding symmetry is broken. In the presence of the background of $\chi$ particles, the mass gets "dressed," $m_{\mathrm{eff}}^{2}=-\mu^{2}+g\left\langle\chi^{2}\right\rangle$. If the field variances are sufficiently large, the symmetry is restored (and is broken when $\left.\left\langle\chi^{2}\right\rangle \leqslant \mu^{2} / g\right)$.

If anomalous correlators are negligible, the field variances can be calculated using expression

$$
\operatorname{var}(\chi) \equiv\left\langle\chi^{2}\right\rangle-\langle\chi\rangle^{2}=\int \frac{d^{d} k}{(2 \pi)^{d}} \frac{n_{k}}{\omega_{k}}
$$

With the help of the self-similar substitution, Eq. (29), we find

$$
\operatorname{var}(\chi, \tau)=A^{\gamma-d+\alpha} \operatorname{var}_{0}(\chi)
$$

Here, the left hand side is taken at conformal time $\eta$, while $\operatorname{var}_{0}(\chi)$ on the right hand side is the variance at some earlier time $\eta_{0}$.

\section{Relativistic regime}

a. Free turbulence. In this case $\gamma=d+\alpha$, see Eq. (41), and we find with $\alpha=1$

$$
\operatorname{var}(\chi, \tau)=A^{2 \alpha} \operatorname{var}_{0}(\chi)=\tau^{-2 p_{i}} \operatorname{var}_{0}(\chi)
$$

For systems that we have studied numerically, $p_{i}=1 / 5$ at early times which span the integration period. Therefore, in the free turbulence regime, we should expect $\operatorname{var}(\chi, \tau)$ $=\tau^{-2 / 5} \operatorname{var}_{0}(\chi)$. This is in agreement with the results of our numerical integration, see Fig. 1. For late-time evolution, when 4-particle interactions will start to dominate, we predict a slower decrease of the variance, $\operatorname{var}(\chi, \tau)$ $=\tau^{-2 / 7} \operatorname{var}_{0}(\chi)$.

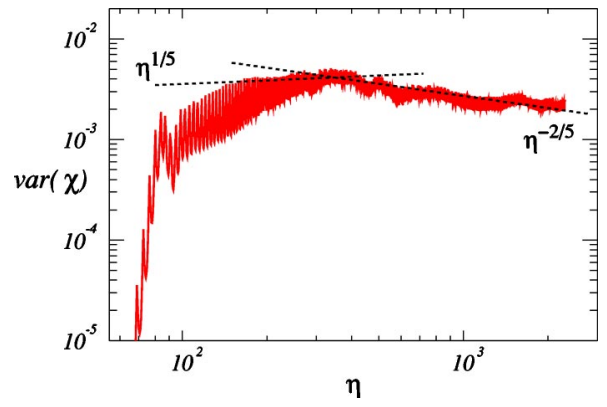

FIG. 10. Time dependence of the variance of $\chi$ field in the model with $h=10 g$ considered in Sec. V.

Note that these results have to be divided by the scale factor squared if expansion of the Universe is important.

b. Driven turbulence. In the regime of stationary turbulence without sink, according to Eq. (45), $\gamma=s$, which gives $\operatorname{var}(\chi, \tau)=A^{s-d+\alpha} \operatorname{var}_{0}(\chi)$. Using Eq. (60), we find $s-d$ $+\alpha=1 /(1-m)$ and

$$
\operatorname{var}(\chi, \tau)=\tau^{-p_{t} /(1-m)} \operatorname{var}_{0}(\chi)=\tau^{p_{i}} \operatorname{var}_{0}(\chi)
$$

where relation Eq. (47) was also used. Therefore, during driven turbulence the variance should grow as $\operatorname{var}(\chi, \tau)$ $=\tau^{1 / 5} \operatorname{var}_{0}(\chi)$ in the models which we have integrated numerically. This is indeed the case as Fig. 10 shows. The transitional period from the regime of parametric resonance to the regime of stationary turbulence at $\eta \sim 10^{2}$ is slightly more pronounced in Fig. 10 as compared to Fig. 6. This may be explained by the fact that different regions of momentum space are emphasized in $\rho_{\chi}$ and in $\operatorname{var}(\chi)$.

\section{Nonrelativistic regime}

In the case of free turbulence we have $\gamma=d$, and in Eq. (84) we have to substitute $\alpha=0$, which corresponds to $\omega_{k}$ $\rightarrow M$ in Eqs. (40), (83). Therefore, $\operatorname{var}(\chi, \tau)=$ const. For driven turbulence we have $\gamma=s=d$, see Eq. (61), and Eq. (84) again gives $\operatorname{var}(\chi, \tau)=$ const.

We see that in the regime of driven turbulence variances are slowly changing functions of time $\left[\propto \tau^{1 / 5}\right.$ in the relativistic case and $\operatorname{var}(\chi, \tau)=$ const in the nonrelativistic case], while energy in particles grows fast, $\rho_{\chi} \propto \tau$ in this regime. This is in accord with the fact that variances can be large right after the initial parametric resonance stage, while the amount of energy transferred during this stage is low and all energy transfers occur in the regime of driven turbulence.

\section{B. Thermalization in the absence of zero mode}

We now apply the results obtained earlier in this paper to the general problem of thermalization of relativistic and nonrelativistic scalar particles, both in Minkowski space-time and in expanding Friedmann universe. We do not restrict ourselves to the models which were studied numerically. Our analysis will be based on expression (29) with the factor $A(\tau)$ being specified for a particular modeled. This expression describes a self-similar propagation of the distribution functions into the ultraviolet. In a classical theory this evo- 
lution continues without bound (unless we consider a nonrelativistic theory in expanding universe).

The classical evolution stops when a system reaches the quantum regime where it can relax to the Bose-Einstein distribution. We adopt that this happens when in a region of momenta, $k_{f}$, which saturate the energy integral, the occupation numbers became of order 1 . In this subsection we consider the case of free turbulence. Then, one can estimate $k_{f}$ using energy conservation and approximating the energy density as $\rho \sim \omega\left(k_{f}\right) k_{f}^{3}$ in the region where $n_{k} \sim 1$. On the other hand, initially the energy was deposited into particles with lower momenta, denoted below as $k_{i}$. The relation $k_{i}$ $\sim M_{\phi}$, where $M_{\phi}$ is the inflaton mass, determines the scale of initial momenta. Eq. (39) gives for the time needed to thermalize a system:

$$
\tau^{\mathrm{th}} \sim\left(k_{f} / k_{i}\right)^{1 / p} .
$$

Actually this should be considered as a lower limit on the thermalization time since we have to add a time which the system will spend in the quantum regime.

As an idealization of the thermalization process we consider the evolution of a subsystem of excitations of a field $\chi$, assuming that a fixed part of energy was deposited into it initially, while since then $\chi$ evolves as an isolated system. In this subsection, for estimates of the thermalization time we neglect the presiding regimes of parametric resonance and of stationary turbulence, since they are much shorter if the relevant coupling constants are not drastically different. We consider the possibility of (partial) thermalization in the regime of driven turbulence in the following subsection.

As a first step we will find the thermalization time which follows from the exact self-similar solutions obtained above. Then we will show that in all cases which we consider, the result coincide, parametrically, with the "naive" perturbative estimates. Doing this comparison we neglect all numerical coefficients.

\section{Relativistic regime}

Equation (87) gives

$$
\tau^{\text {th }} \sim\left(\rho_{f}^{1 / 4} / M_{\phi}\right)^{1 / p} .
$$

The expansion of the Universe is easily treated in conformal reference frame. We have $\rho_{f}=\rho_{i}=c_{\chi} \rho_{\text {tot }}$, where $c_{\chi}$ is the fraction of the inflaton energy deposited into the field $\chi$ during preheating and driven turbulence. This finalizes the answer. The result is general and is valid for any model. The initial inflaton energy can be written as $\rho_{\text {tot }} \sim k_{i}^{2} \phi_{0}^{2}$ $\sim M_{\phi}^{2} M_{\mathrm{Pl}}^{2}$, where $\phi_{0}$ is the initial amplitude of inflaton oscillations. We find with $p=1 / 7$, Eq. (65), which corresponds to a relativistic theory with dimensionless couplings

$$
\tau^{\mathrm{th}} \sim c_{\chi}^{7 / 4}\left(\frac{M_{\mathrm{Pl}}}{m_{\phi}}\right)^{7 / 2} \sim c_{\chi}^{7 / 4} 10^{21} .
$$

We used here the inflaton parameters, $M_{\phi} \approx 10^{-6} M_{\mathrm{Pl}}$ in $M_{\phi}^{2} \phi^{2}$ model, or $M_{\phi}=\sqrt{\lambda} M_{\mathrm{Pl}}$ with $\lambda \approx 10^{-13}$ in the $\lambda \phi^{4}$ inflationary model. To avoid confusion, note that the definition of $\tau$ is different in different models since it involves $t_{0}$ $\sim \Gamma^{-1}$.

We can assume $c_{\chi} \approx 1$ if the Universe expansion can be neglected (this may be of interest for problems outside of inflationary cosmology), and if the number of competing channels (other fields beyond $\chi$ to which the initial energy can be deposited) is not large.

a. Minkowski space-time. Let us show that Eq. (88) agrees with the "naive" perturbative estimate. For this estimate we define $\tau$ as $\tau=t_{R} \Gamma$, where $t_{R}$ is the perturbative estimate of the thermalization time $t_{R}^{-1} \sim \sigma n$. In the $\lambda \phi^{4}$ model $\sigma$ $\sim \lambda^{2} / T^{2}, n \sim T^{3}, T \sim k_{f}$ and therefore $t_{R}^{-1} \sim \lambda^{2} k_{f}$. On the other hand, parametric resonance stops when the rate of rescattering from the resonance band becomes equal to the rate of particle production $\mu \sim M_{\phi} \sim k_{i}$. This gives $\Gamma \sim k_{i}$ and we find $\tau \sim \Gamma t_{R} \sim k_{i} / \lambda^{2} k_{f}$. Now, $\rho \sim k_{f}^{4}$ and $\rho \sim k_{i}^{4} n_{k}$, where $n_{k}$ correspond to the typical occupation numbers at the time when parametric resonance stops, $n_{k} \sim 1 / \lambda$. We obtain $t_{R} \Gamma$ $=\left(\rho^{1 / 4} / k_{i}\right)^{7}$, in agreement with Eq. (88).

$b$. Friedmann universe. In this case we can estimate the final temperature as $T \sim k_{f} / a(\tau)$. Let us consider a radiation dominated universe with $a(\tau)=H_{0} \eta_{0}(\tau-1)+1$, see Eq. (68). We neglect the rapid epoch of stationary turbulence, and $\eta_{0}$ corresponds to a time when the evolution of $\chi$ is driven by its self-interaction with self-coupling $\lambda_{\chi}$, i.e. $\eta_{0}^{-1} \sim \Gamma \sim \lambda_{\chi}^{2} n_{k}^{2} k_{i} \sim \lambda_{\chi}^{2}\left(c_{\chi} \rho_{\text {tot }}\right)^{2} / k_{i}^{7}$, where we have used $\rho_{\chi}$ $\sim k_{i}^{4} n_{k}$. On the other hand $H_{0} \sim \rho_{\text {tot }}^{1 / 2} / M_{\mathrm{Pl}}$. Combining this with Eq. (88) we find $a(\tau)=H_{0} \eta_{0} \tau \sim c_{\chi}^{-1 / 4} \rho_{\text {tot }}^{1 / 4} / \lambda_{\chi}^{2} M_{\mathrm{Pl}}$. For the final thermalization temperature we obtain $T \sim k_{f} / a(\tau)$ $\sim c_{\chi}^{1 / 2} \lambda_{\chi}^{2} M_{\mathrm{Pl}}$, where we have used $k_{f} \sim \rho_{\chi}^{1 / 4}$. This again agrees with the naive estimate, $\sigma n \sim H$.

Numerically, $T \sim \lambda_{\chi}^{2} M_{\mathrm{Pl}} \sim 100 \mathrm{eV}$, if we use the strength of the inflaton self-coupling, $\lambda \approx 10^{-13}$. Therefore, in a realistic model, at least some couplings should be significantly larger than this scale.

\section{Nonrelativistic regime}

Now we consider the turbulent evolution of $X$ particles of mass $M_{X}$ towards thermalization in the nonrelativistic regime. A nonrelativistic regime should not be essential for thermalization of the Universe after inflation. However, depending on parameters, nonrelativistic particles with large occupation numbers may be present during the initial stages of thermalization. This happens e.g. at large values of the resonance parameter for $\mathrm{X}$ particles which are heavier than the inflaton. It is interesting and important to trace the evolution of such particles until distributions either "freeze out" due to Universe expansion, or enter a relativistic regime, or reach the quantum domain and may approach thermal distributions. Interestingly, we found that the latter situation may also be realized after inflation for large values of couplings.

We assume that the relaxation is due to the self-interaction $\lambda_{X} X^{4}$. The particle number conserves in the conformal reference frame in this regime, and Eq. (87) gives

$$
\tau^{\mathrm{th}}=c_{X}^{1 / 3 p}\left[\frac{1}{M_{\phi}}\left(\frac{\rho_{f}}{M_{X}}\right)^{1 / 3}\right]^{1 / p},
$$


where $c_{X}$ is the fraction of the inflaton energy which initially was deposited into the field $X$ (this fraction should be measured at the time when the self-similar evolution starts). In the present case $p=1 / 2$, see Eq. (59), and similarly to Eq. (89) we find for the relaxation time

$$
\tau \sim\left(c_{X} \frac{M_{\mathrm{Pl}}^{2}}{M_{\phi} M_{X}}\right)^{2 / 3} \sim\left[c_{X} \frac{M_{\phi}}{M_{X}}\right]^{2 / 3} 10^{8} .
$$

In this expression $M_{\phi} / M_{X} \sim 1$ since Bosons which are much heavier than the inflaton are not created, and in the opposite regime $X$ particles would have been relativistic.

As we have seen in Sec. IV C 2 there is no real relaxation of massive particles when the expansion has become important. If some relaxation happens, it should occur during the time interval when the scale factor does not deviate significantly from its initial value. Then the expansion can be neglected and the relaxation proceeds as in Minkowski spacetime. Let us show that the expressions above agree with the "naive" perturbative estimate in the latter case.

a. Minkowski space-time. The perturbative relaxation time in the final state can be estimated as $t_{R}^{-1} \sim v \sigma n$, where $\sigma$ $\sim \lambda_{X}^{2} / M_{X}^{2}$ and $n \sim k_{f}^{3}$. Therefore $t_{R}^{-1} \sim \lambda_{X}^{2} k_{f}^{4} / M_{X}^{3}$. On the other hand, the rate in the initial state is given by a similar expression, but is multiplied by large occupation numbers in the initial state [89] (which can be viewed as Boseamplification factor), $\Gamma \sim v \sigma n n_{k} \sim \lambda_{X}^{2} n^{2} / k_{i}^{2} M_{X}^{3}$, where we used $n \sim k_{i}^{3} n_{k}$. We obtain $t_{R} \Gamma \sim n^{2} / k_{f}^{4} k_{i}^{2} \sim\left(\rho / M_{X}\right)^{2 / 3} / k_{i}^{2}$, where $\rho=M_{X} n$. This agrees with Eq. (90).

$b$. Friedmann universe. To estimate the thermalization time and temperature we need to know the typical rate of reactions and the value of the Hubble parameter at the beginning of self-similar evolution. For definiteness we consider the situation which arises after preheating in the massive inflaton model coupled to a heavy field $X$. We assume that self-coupling of the $X$ field is sufficiently large, such that the "parametric" decay of the inflaton is halted by $X$ rescattering on each other. Using results of Ref. [9] we conclude that at the moment when the inflaton zero mode decays completely, the energy density in the $X$ field can be estimated as $\rho_{\chi} \sim M_{X}^{4} / \lambda_{\chi}$, while the rate of re-scattering is $\eta_{0}^{-1} \sim M_{X}$. This gives $H_{0} \eta_{0} \sim \lambda_{\chi}^{-1 / 2} M_{X} / M_{\mathrm{Pl}} \sim q^{-1 / 2}$, where $q$ is the initial resonance parameter. Since $q$ can be very large, the product $H_{0} \eta_{0}$ can be small and the expansion is not significant at the initial stage of the self-similar evolution. On the other hand, the time needed to reach the quantum regime is of order $\tau^{t h} \sim\left(k_{f} / k_{i}\right)^{1 / p}$. Since the particle number conserves during the period of self-similar evolution we have $k_{f}^{3}$ $\sim M_{X}^{3} / \lambda_{\chi}$, while $k_{i} \sim M_{X}$ at the end of parametric resonance stage. This gives $\tau^{t h} \sim\left(1 / \lambda_{\chi}\right)^{1 / 3 p} \sim \lambda_{\chi}^{-2 / 3}$. The condition $H_{0} \eta_{0} \tau^{\text {th }}<1$ gives $\lambda_{\chi}^{7 / 6}>M_{X} / M_{\mathrm{Pl}}$ as a necessary condition to reach a thermal state before the freezeout of distribution functions. Using inflationary normalization, we conclude that nonrelativistic particles created in "parametric resonance" have a chance to thermalize between themselves in an expanding universe if $\lambda_{\chi}>10^{-5}$.

\section{A faster route to thermalization?}

Considering the regime of free turbulence, we have obtained estimates for the thermalization time which are in agreement with "naive" perturbation theory. It was important in these estimates that the relativistic free turbulence propagates with $p=1 / 7$. This should be true at sufficiently late times, when all effects related to zero mode become insignificant. However, free turbulence driven by 3-particle interactions in the presence of a zero mode evolves with $p$ $=1 / 5$. The evolution of the front of particle distributions is even faster in the case of driven turbulence, when $p=2 / 5$. If the quantum domain is already reached during one of these two stages our estimates for thermalization should be changed.

Here we consider the question whether a subsystem of $\chi$ particles can reach the quantum region in the regime of a stationary turbulence.

\section{Driven turbulence}

The quantum domain is reached in the regime of driven turbulence if the power law of the inertial range will extend up to $n_{k} \sim 1$. In other words, $n_{k}=\left(k / k_{T}\right)^{-s}$ should be valid up to $k=k_{T}$. Let us consider the model were the largest coupling is the self-coupling of the $\chi$ field. The normalization of $n_{k}$ can be fixed if we recall that in the region of the source, $k \sim k_{i}$, the $\chi$-particle distribution is given by $n_{\chi}$ $\sim 1 / \lambda_{\chi}$. This gives $k_{T} \sim k_{i} \lambda_{\chi}^{-1 / s}$, or the time needed to reach the quantum region is given by

$$
\tau \sim \lambda_{\chi}^{-1 / s p},
$$

where we have used Eq. (87).

On the other hand, the energy in the subsystem of $\chi$ particles grows in the regime of driven turbulence as $\rho_{\chi}(\tau)$ $=\tau \rho_{\chi}(1)$, and should not exceed the total energy stored in the inflaton zero-mode oscillations. The initial energy can be estimated as $\rho_{\chi}(1) \sim k_{\text {res }}^{4} n_{\chi}$, where $k_{\text {res }} \sim q^{1 / 4} \omega_{\phi}$ and $q$ is the resonance parameter: $q=\lambda_{\phi \chi} \Phi_{0}^{2} / M_{\phi}^{2}$ in the $M_{\phi}^{2} \Phi^{2}$ inflaton model, or $q=\lambda_{\phi \chi} / \lambda_{\phi}$ in the $\lambda \Phi^{4}$ inflaton model. This gives $\rho_{\chi}(1) / \rho_{\text {tot }} \sim \lambda_{\phi \chi} / \lambda_{\chi}$, and we obtain the bound

$$
\tau<\lambda_{\chi} / \lambda_{\phi \chi} .
$$

We conclude that the quantum domain can be reached in the regime of driven turbulence if $\lambda_{\chi}>\lambda_{\phi \chi}^{s p /(s p+1)}=\lambda_{\phi \chi}^{3 / 8} \sim 10^{-4}$. Here we have used $s=3 / 2, p=2 / 5$ and $\lambda_{\phi \chi} \sim 10 \lambda_{\phi}$. These values are realistic, therefore, physical implications of driven turbulence in applications to thermalization deserve further study.

\section{CONCLUSIONS}

We have studied the process of thermalization of classical systems, which at some point in their evolution are in a highly nonequilibrium state with energy being concentrated in a deep "infrared" region of momenta. Such states naturally appear e.g. during reheating of the Universe after cosmological inflation. We have shown that the process of re- 
laxation in such systems can be divided, in the general case, into three distinct stages.

In the models of the type we have considered in this paper, the initial stage of preheating [4] is powered by parametric resonance. During this initial linear stage the rate of energy transfer is the fastest. The energy in particles grows exponentially. However, in the physical situation of reheating after inflation, the coupling constants have to be sufficiently large to insure an acceptably short time-scale of the subsequent thermalization, while with large couplings, only a negligible fraction of the initial inflaton energy is transferred into fluctuations during the parametric resonance stage $[9,10]$.

We have shown that in such situations the linear stage is followed by the regime of a driven stationary turbulence. During this stage, the energy in particles grows linearly in time. The regime of stationary turbulence stops as soon as the energy in particles starts to dominate the overall energy balance. Therefore, this regime is a major mechanism of energy transfer from the oscillating inflaton zero mode into other species in realistic models of the type we have considered here. This period of evolution is also prompt. It should be noted that the source which drives the turbulence is powerful because coherence effects are still strong in the relevant region of momenta.

The subsequent long stage of thermalization classifies as free turbulence. This stage should be generic. The energy in particles is conserved during this epoch, while the shape of the particle distribution function changes in a self-similar way with the front of the distribution propagating into the ultraviolet. This stage continues until the quantum regime is reached and particles can relax to Bose-Einstein distributions. Applying conventional kinetic theory we have calculated analytically the time needed to equilibrate a system and the resulting temperature in terms of coupling constants and initial inflaton amplitude. The result coincides parametrically with the "naive" perturbative estimates [1].

We made a comparison of kinetic theory with the numerical integration of scalar field models on the lattice. We show that, at late times, the kinetic approach is applicable, resulting in a weak wave turbulence regime [69]. In the models considered numerically, the evolution is driven by threeparticle scattering in the background of zero-mode oscillations. The characteristic exponents calculated within the framework of wave kinetic theory are consistent with the results of our lattice simulations.

\section{ACKNOWLEDGMENTS}

We are grateful to S. Khlebnikov, A. Linde, A. Riotto, V. Rubakov, C. Schmid, D. Semikoz, and M. Shaposhnikov for many useful discussions during various stages of this project. R.M. thanks the Tomalla Foundation for financial support.

\section{APPENDIX A: NUMERICAL PROCEDURE}

In our study we have developed and employed a higher accuracy version of the LATTICEEASY code [90]. Various correlation functions were measured with the use of fast Fourier transform (FFT), adopted from Numerical Recepies [91]. Essential details of our procedure can be found in Refs. $[7,9,25,90]$. Here we describe specific choices of parameters, significant important differences in the integration scheme and give exact definitions of lattice observables.

The numerical integration was done on a 3D cubic lattice with periodic boundary conditions. The lattice is parametrized by the box size $L$, and the number of lattice points per dimension, $N$. These give the lattice spacing $b \equiv L / N$ and the total number of lattice sites, $N^{3}$ in three dimensions.

The results presented in the paper are taken from simulations with $256^{3}$ lattice sites and a box size $L$ chosen to fit a particular problem. For example, in the case of Eq. (3), $L$ $=7.5 \pi$. With this box size the infrared modes which belong to the resonance band are still well represented, while the ultraviolet lattice cutoff is sufficiently far away from the occupied modes, therefore the particle spectra are not distorted even at late times. We have studied the dependence of our results on the lattice and box size to avoid lattice artifacts.

The finite-differences scheme that was used is 2 nd order in time and 4th order in space.

\section{Finite-differences scheme}

We write the equations of motion (3) or (78), (79) as fourth order finite differences on a three-dimensional spatial cubic lattice with periodic boundary conditions. The corresponding equations were evolved with the use of a symplectic integration scheme. Details are as follows.

Particle wave numbers are discrete on the lattice, $\boldsymbol{k}$ $=\left(n_{1}, n_{2}, n_{3}\right) k_{0}$, where $-N / 2 \leqslant n_{j} \leqslant N / 2$ and $k_{0}=(2 \pi) / L$. The phase space is restricted to $k_{0} \leqslant k \leqslant k_{\max }$, where $k_{\max }$ $=\sqrt{3} k_{0} N / 2$. To avoid distortion at high momenta, it is desirable to take large $\mathrm{N}$. This, however, is limited by the capabilities of the computer used. The choice of small values for $L$ is also prohibited since that will lead to infrared distortions and may even move the resonance band out of the integration box. The problem is alleviated by the choice of a finitedifferences scheme which is fourth order in space. This can be quantified in the following way.

The lattice realization of the Laplacian in our scheme is given by

$$
\begin{aligned}
\triangle_{\mathrm{L}} \Phi(\boldsymbol{x})= & \frac{1}{b^{2}} \sum_{\boldsymbol{e}}\left[-\frac{1}{12} \Phi(x+2 b \boldsymbol{e})+\frac{4}{3} \Phi(x+b \boldsymbol{e})\right. \\
& \left.-\frac{5}{2} \Phi(x)+\frac{4}{3} \Phi(x-b \boldsymbol{e})-\frac{1}{12} \Phi(x-2 b \boldsymbol{e})\right] .
\end{aligned}
$$

The vector index $\boldsymbol{e}$ runs over the three orthonormal directions of the lattice. $\triangle_{\mathrm{L}}$ is a fourth order approximation, i.e. ( $\triangle$ $\left.-\triangle_{\mathrm{L}}\right) \Phi(\boldsymbol{x}) \sim \mathcal{O}\left(b^{4}\right)$ for a differentiable function $\Phi(\boldsymbol{x})$. The Fourier transform of $\triangle_{\mathrm{L}}$ differs from that of $\triangle$, which would be given by multiplication with $k^{2}$. Therefore the dispersion relation for a massless field on the lattice is also different, and is given by 


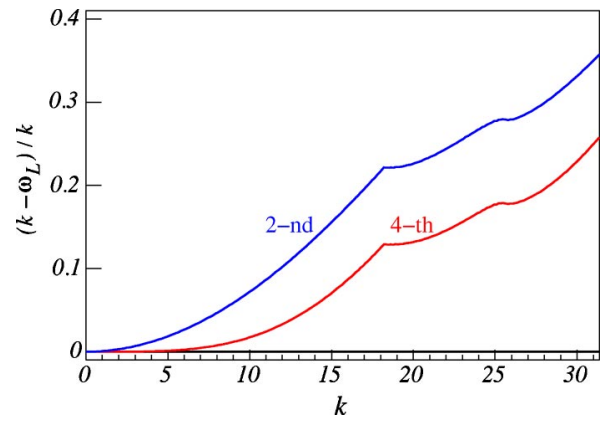

FIG. 11. Deviation of the dispersion law for massless excitations on the lattice, $\left(k-\omega_{L}\right) / k$, for second and fourth order finitedifference schemes.

$$
\omega_{\mathrm{L}}^{2}(k)=\frac{1}{b^{2}} \sum_{i=1}^{3}\left(\frac{5}{2}-\frac{8}{3} \cos \left(b k_{i}\right)+\frac{1}{6} \cos \left(2 a k_{i}\right)\right) .
$$

We find that $\omega_{\mathrm{L}}^{2} \leqslant k^{2}$ and $\omega_{L}^{2}-k^{2} \sim \mathcal{O}\left(k^{6} b^{4}\right)$ for small $k$. Numerically, for $k \leqslant k_{\max } / 3$, the relative difference between $k$ and $\omega_{L}$ is less then a percent, while for larger $k$ it grows up to about $30 \%$ difference at $k=k_{\max }$. This means, that we can expect essentially undistorted self-similar and turbulent solutions on the lattice, if the dominating modes have wave vectors $k \leqq k_{\max } / 3$. In the case of a second order realization of $\triangle_{\mathrm{L}}$, we find a considerably smaller available phase space, $k \lesssim k_{\max } / 10$. This is illustrated in Fig. 11 where we plot $(k$ $\left.-\omega_{L}\right) / k$ as a function of $k$ for the second and fourth order calculation schemes on the lattice $L=7.5 \pi$, used in our integration of the problem Eq. (3). We see that up to $k=10$, which essentially encompasses the support region of the distribution functions, see Fig. 3, the dispersion law on the lattice represents the continuum correctly. That is why selfsimilarity was not distorted on our lattice and could have being detected. (The small deviations from self-similarity, which can be observed at the very tail of the distribution and at the latest time, see Fig. 3, are caused by the distortion of the dispersion law which starts to be non-negligible here.)

\section{Classical approximation and stochastic initial conditions}

The initial linear stage of parametric resonance has a complete quantum description, which is best expressed in the language of Bogoliubov transformations. The quantum description of this linear problem can be mapped into an equivalent classical problem [7]. In our dimensionless variables the initial conditions for the classical description are given by the following probability distribution for field fluctuations in Fourier space:

$$
\begin{aligned}
\mathbf{P}[\psi, \dot{\psi}] \sim & \exp \left\{-\frac{2}{\lambda_{\Phi}} \int d^{3} k 2 \omega_{k}^{\psi}\left(\tau_{0}\right)\left|\psi_{k}\right|^{2}\right\} \\
& \times \delta^{F}\left[\dot{\psi}_{\boldsymbol{k}}+i \omega_{k}^{\psi}\left(\tau_{0}\right) \psi_{k}\right] .
\end{aligned}
$$

Here " $\psi$ " should be replaced by one of the fields $\phi$ or $\chi$ that are the dynamical variables in the simulated equations (3) or (78), (79), and $\omega_{k}^{\phi}\left(\eta_{0}\right) \simeq \sqrt{k_{L}^{2}+3}$, while $\omega_{k}^{\chi}\left(\eta_{0}\right) \simeq \sqrt{k_{L}^{2}+g}$.
The symbol $\delta^{F}(\ldots)$ is a functional Dirac distribution so that the canonical momenta are locked to the classical trajectory.

\section{Measured quantities}

We measure various physical quantities both in configuration space and in Fourier space. In configuration space it is convenient to measure the zero mode, $\phi_{0} \equiv\langle\phi\rangle$, and the variance, $\operatorname{var}(\phi) \equiv\left\langle\phi^{2}\right\rangle-\phi_{0}^{2}$. In Fourier space we measure particle number and other correlators.

For large $N$ lattice averages basically coincide with the statistical ones (ergodic theorem). We use this fact to measure expectation values (zero modes), variances and higher cumulants of fields and their conjugate momenta.

a. Spatial lattice averages. For averages defined in configuration space $\langle\mathcal{O}\rangle \equiv V^{-1} \int d^{3} x \mathcal{O}$, which on the lattice is expressed as the sum over the lattice points $\langle\mathcal{O}\rangle$ $\equiv N^{-3} \sum_{i} \mathcal{O}_{i}$.

b. Fourier spectra. For monitoring purposes we make a FFT transform at least every period of inflaton oscillation. The wave amplitudes of fourier transformed fields are defined by Eq. (B20), see Appendix B. In the dimensionless units that we use in the numerical simulation the physical wave amplitudes take the form

$$
\tilde{a}_{k}^{\psi} \equiv \frac{1}{\sqrt{\lambda_{\Phi}}} \frac{\omega_{k}^{\psi} \psi_{k}+i \dot{\psi}_{k}}{(2 \pi)^{3 / 2} \sqrt{2 \omega_{k}^{\psi}}}
$$

where again " $\psi$ " stands for the dynamical variables $\phi$ or $\chi$ in Eqs. (3) or (78), (79). The dimensionless frequencies are given by $\omega_{k}^{\psi} \equiv \sqrt{k_{L}^{2}+m_{\mathrm{eff}}^{\psi / 2}}$, where $m_{\mathrm{eff}}^{\phi 2}=3\left\langle\varphi^{2}\right\rangle+g\left\langle\chi^{2}\right\rangle$ and $m_{\text {eff }}^{\chi}=g\left\langle\varphi^{2}\right\rangle+3 h\left\langle\chi^{2}\right\rangle$. Making use of $a_{k}$, we calculate various correlators, $\quad n(k) \equiv\left\langle a_{k}^{*} a_{k}\right\rangle, \quad \sigma(k) \equiv\left\langle a_{k} a_{-k}\right\rangle$, $\left\langle a^{*} a^{*} a a\right\rangle$, etc. The first one, which corresponds to the particle occupation numbers, is of prime interest.

Note that with this simple definition of quasiparticles the Hamiltonian is not diagonal in terms of $a_{k}^{\chi}$ and $a_{k}^{\phi}$ wave amplitudes if interaction energy is important. Therefore, the related definition of e.g. particle number is good only for modes with dominating kinetic energy.

\section{APPENDIX B: KINETIC EQUATION FOR CLASSICAL WAVES}

Following the general approach of Refs. $[69,81]$ we derive the wave kinetic equation for the classical system of interest, the massive $\lambda \phi^{4}$ theory in $d$ dimensions with Hamiltonian density

$$
\mathcal{H}=\frac{1}{2} \dot{\phi}^{2}+\frac{1}{2}(\nabla \phi)^{2}+\frac{M^{2}}{2} \phi^{2}+\frac{\lambda}{4} \phi^{4},
$$

and in the presence of an oscillating classical background. We assume random wave fields which are statistically uniform, i.e. the equal time correlation functions of $\phi$ and its canonical conjugate momentum $\dot{\phi}$ are homogeneous and isotropic. We also assume the field to be weakly interacting. 
The first step in the derivation of the kinetic equation for an arbitrary system is to find Fourier wave amplitudes, $a_{k}$, such that the quadratic part of the Hamiltonian is diagonal in $a_{k}$, i.e.:

$$
H_{2}=\int d^{d} k \omega_{k} a_{k}^{*} a_{k}
$$

The general equation of motion for the wave amplitudes is

$$
\frac{d a_{\mathbf{k}}}{d t}=\frac{\partial a_{\mathbf{k}}}{\partial t}-i \omega_{k} a_{\mathbf{k}}-i \frac{\delta H_{i n t}}{\delta a_{\mathbf{k}}^{*}}
$$

where $H_{\text {int }} \equiv H-H_{2}$. The first term on the left-hand side is due to a possible explicit time-dependence in the definition of $a_{k}$, which can appear for example in case of a timevarying background.

In the kinetic approach we want to get rid of rapidly varying phases of the wave amplitudes, i.e. to derive the equation for the slowly changing "occupation numbers," $n_{k} \sim a_{k}^{*} a_{k}$. To achieve this we multiply Eq. (B3) by $a_{k}^{*}$, add the complex-conjugate expression and average. The result will contain higher order correlators induced by interaction terms. The resulting BBGKY-hierarchy of equations for Fourier cumulants can be solved, e.g., in the random phase approximation in consistent perturbative expansion.

In the case of Eq. (B1) the wave amplitudes for the fluctuation fields are solutions of

$$
\begin{aligned}
\delta \phi_{\boldsymbol{k}} & =\frac{(2 \pi)^{d / 2}}{\sqrt{2 \omega_{k}}}\left(a_{\boldsymbol{k}}+a_{-\boldsymbol{k}}^{*}\right), \\
\delta \dot{\phi}_{\boldsymbol{k}} & =\frac{(2 \pi)^{d / 2} \sqrt{\omega_{k}}}{\sqrt{2} i}\left(a_{\boldsymbol{k}}-a_{-\boldsymbol{k}}^{*}\right)
\end{aligned}
$$

where $\delta \phi_{k}$ and $\delta \dot{\phi}_{k}$ are Fourier transforms of the canonical field and of its conjugate momenta respectively, shifted by the "zero mode" $\phi_{0}=\langle\phi\rangle$ and $\dot{\phi}_{0}=\langle\dot{\phi}\rangle$. This gives

$$
a_{k} \equiv \frac{\omega_{k} \delta \phi_{k}+i \delta \dot{\phi}_{k}}{(2 \pi)^{d / 2} \sqrt{2 \omega_{k}}} .
$$

From the start we include in $\omega_{k}$ the interaction with the bath of fluctuations,

$$
\omega_{k}^{2}=k^{2}+M^{2}+3 \lambda \phi_{0}^{2}+3 \lambda\left\langle\delta \phi^{2}\right\rangle,
$$

i.e. $a_{k}$ correspond to "quasiparticles." The second order correlators in homogeneous and isotropic background should be "diagonal",

$$
\begin{aligned}
\left\langle a_{\boldsymbol{k}}^{*} a_{\boldsymbol{q}}\right\rangle & =n_{k} \delta^{(d)}(\boldsymbol{k}-\boldsymbol{q}), \\
\left\langle a_{\boldsymbol{k}} a_{\boldsymbol{q}}\right\rangle & =\sigma_{k} \delta^{(d)}(\boldsymbol{p}+\boldsymbol{q}) .
\end{aligned}
$$

\section{Microscopical equations of motion}

We derive equations of motion for the zero mode and for wave amplitudes starting from

$$
\square \phi+M^{2} \phi+\lambda \phi^{3}=0 .
$$

a. Zero mode. Averaging Eq. (B10) we obtain

$$
\ddot{\phi}_{0}+\left(M^{2}+3 \lambda\left\langle\delta \phi^{2}\right\rangle\right) \phi_{0}+\lambda \phi_{0}^{3}+\lambda\left\langle\delta \phi^{3}\right\rangle=0 .
$$

In this equation $\left\langle\delta \phi^{3}\right\rangle$ is small compared to the other terms and may be neglected locally in time in a state which is close to a Gaussian. If additionally $\left\langle\delta \phi^{2}\right\rangle$ is either weakly varying or small compared to all other terms in Eq. (B11) the solution is given by the Jacobian cosine cn $[79,92,93]$

$$
\phi_{0}(t) \simeq \bar{\phi}_{0} \quad \operatorname{cn}\left(\mu t, \frac{1}{\sqrt{2}} \frac{\lambda \bar{\phi}_{0}}{\mu}\right),
$$

where $\bar{\phi}_{0}$ is the amplitude and

$$
\mu \equiv \sqrt{\lambda \bar{\phi}_{0}^{2}+3 \lambda\left\langle\delta \phi^{2}\right\rangle+M^{2}} .
$$

The period of this function is

$$
T_{0}=4 \mu^{-1} K\left(\frac{1}{\sqrt{2}} \frac{\lambda^{1 / 2} \bar{\phi}_{0}}{\mu}\right),
$$

where $K(y)$ is the complete elliptic integral of the first kind. This defines the effective frequency, $\omega_{c}=2 \pi / T_{0}$. In the large amplitude limit, $\mu=\lambda^{1 / 2} \bar{\phi}_{0}$, we find $\omega_{c} \simeq 0.85 \mu$. For arbitrary $\mu$ one can write the following decomposition:

$$
\omega_{c} \simeq \mu-\frac{1}{8} \frac{\lambda \bar{\phi}_{0}^{2}}{\mu}
$$

which is fairly accurate, the maximum deviation from the exact expression is $3 \%$ at $\mu=\lambda^{1 / 2} \bar{\phi}_{0}$. For small amplitude of zero-mode oscillations, this expression can be further approximated as

$$
\omega_{c} \simeq M_{\mathrm{eff}}\left(1+\frac{3 \lambda \bar{\phi}_{0}^{2}}{8 M_{\mathrm{eff}}^{2}}\right)
$$

where $M_{\mathrm{eff}} \equiv\left(M^{2}+3 \lambda\left\langle\delta \phi^{2}\right\rangle\right)^{1 / 2}$. This deviates from exact result by less than $4 \%$ at $\lambda \bar{\phi}_{0}<M_{\text {eff }}$.

For a general discussion of the kinetic equations in the background of a zero mode it might be useful to expand $\phi_{0}(t)$ in a Fourier series. However, this decomposition for the elliptic Jacobi function is strongly dominated by the first harmonic with $\omega=\omega_{c}$. Even at $\mu=\lambda^{1 / 2} \bar{\phi}_{0}$, the relative amplitude of the first harmonic is $\approx 0.96$, and it approaches unity with decreasing $\bar{\phi}_{0}$. Therefore, in what follows we will restrict ourselves to the first term in the Fourier decomposition of $\phi_{0}(t)$.

It is useful to define wave amplitudes for the zero mode

$$
a_{c} \equiv \sqrt{2 \omega_{c}} \bar{\phi}_{0} e^{-i \omega_{c} t}
$$

in terms of which the zero mode can be represented as 


$$
\phi_{0}=\frac{a_{c}+a_{c}^{*}}{\sqrt{2 \omega_{c}}} .
$$

One can also introduce an effective occupation number of "condensed waves,"

$$
n_{c}=a_{c}^{*} a_{c}=2 \omega_{c} \bar{\phi}_{0}^{2} .
$$

b. Wave amplitudes. The equations of motion for the wave amplitudes with nonzero momentum can be written as

$$
\dot{a}_{\boldsymbol{k}}=-i \omega_{k} a_{\boldsymbol{k}}+\frac{1}{2} \frac{\dot{\omega}_{k}}{\omega_{k}} a_{-\boldsymbol{k}}^{*}+C_{k}^{(3)}+C_{k}^{(4)},
$$

where

$$
\begin{aligned}
C_{\boldsymbol{k}}^{(3)} \equiv & -3 i \lambda \phi_{0} \int d \Omega_{k 12}\left[\delta \phi_{\boldsymbol{p}_{1}} \delta \phi_{\boldsymbol{p}_{2}}-\left\langle\delta \phi_{\boldsymbol{p}_{1}} \delta \phi_{\boldsymbol{p}_{2}}\right\rangle\right] \\
& \times \delta^{(d)}\left(\boldsymbol{k}-\boldsymbol{p}_{1}-\boldsymbol{p}_{2}\right), \\
C_{\boldsymbol{k}}^{(4)} \equiv & -i \lambda \int d \Omega_{k 123}\left[\delta \phi_{\boldsymbol{p}_{1}} \delta \phi_{\boldsymbol{p}_{2}} \delta \phi_{\boldsymbol{p}_{3}}\right. \\
& \left.-3 \delta \phi_{\boldsymbol{p}_{1}}\left\langle\delta \phi_{\boldsymbol{p}_{2}} \delta \phi_{\boldsymbol{p}_{3}}\right\rangle-\left\langle\delta \phi_{\boldsymbol{p}_{1}} \delta \phi_{\boldsymbol{p}_{2}} \delta \phi_{\boldsymbol{p}_{3}}\right\rangle\right] \\
& \times \delta^{(d)}\left(\boldsymbol{k}-\boldsymbol{p}_{1}-\boldsymbol{p}_{2}-\boldsymbol{p}_{3}\right),
\end{aligned}
$$

and

$$
d \Omega_{k 12} \equiv \frac{d^{d} p_{1} d^{d} p_{2}}{\sqrt{2 \omega_{k}}(2 \pi)^{3 d / 2}}, \quad d \Omega_{k 123} \equiv \frac{d^{d} p_{1} d^{d} p_{2} d^{d} p_{3}}{\sqrt{2 \omega_{k}}(2 \pi)^{5 d / 2}} .
$$

$C_{\boldsymbol{k}}^{(3)}$ describes three wave interactions in the background of a zero mode, while $C_{\boldsymbol{k}}^{(4)}$ corresponds to four wave scattering. The averages in these expressions appeared because, first, we separated the zero mode out of the equation for fluctuations, and, second, we used the effective frequency for quasiparticles, Eq. (B7). Due to this choice the averages of $C^{(a)}$ times $a_{k}$ or $a_{k}^{*}$ will have the structure of cumulants, which in turn will deviate from zero only due to correlations induced by processes of scattering.

Multiplying Eq. (B20) by $a_{k}^{*}$ or by $a_{k}$ and adding the complex-conjugate expressions, we find

$$
\begin{gathered}
\dot{n}_{k}=\frac{\dot{\omega}_{k}}{\omega_{k}} \operatorname{Re} \sigma_{k}+\operatorname{Im} I_{3}(k)+\operatorname{Im} I_{4}(k), \\
i \dot{\sigma}_{k}=2 \omega_{k} \sigma_{k}+\frac{i}{2} \frac{\dot{\omega}_{k}}{\omega_{k}} n_{k}+I_{3}^{*}(k)+I_{4}^{*}(k)
\end{gathered}
$$

where

$$
\begin{aligned}
& I_{3}(k)=6 \lambda \phi_{0} \int d \Omega_{k 12}\left\langle a_{k}^{*} \delta \phi_{p_{1}} \delta \phi_{p_{2}}\right\rangle_{c}, \\
& I_{4}(k)=2 \lambda \int d \Omega_{k 123}\left\langle a_{k}^{*} \delta \phi_{p_{1}} \delta \phi_{p_{2}} \delta \phi_{p_{3}}\right\rangle_{c} .
\end{aligned}
$$

Here $\langle\ldots\rangle_{c}$ denotes the cumulants, which in a diagrammatic language are identified with connected diagrams, see e.g. Ref. [94].

In situations when $I_{3}(k)$ and $I_{4}(k)$ are negligible, Eqs. (B21) and (B22) describe particle creation in a timedependent classical background, or parametric excitation if it is periodic. (Note that the quantum version of these equations at this stage can be obtained by the formal substitution $\left.n_{k} \rightarrow 1 / 2+n_{k} \cdot\right)$

Note the following:

(i) In our case $\omega_{k}$ contains an rapidly oscillating term, due to interaction with the zero mode. However, at late times and at large $k$ it is small. e.g., in our numerical integration in the region of $k$ near the peak of the spectral energy distribution, this term is of order $10^{-3}$, see Figs. 1 and 3. We neglect this term in what follows.

(ii) The coefficient in front of the integral Eq. (B23) is rapidly oscillating. Moreover, oscillations are not harmonic if the amplitude of $\phi_{0}$ is large. Unharmonicity can be dealt with by expanding $\phi_{0}(t)$ in Fourier time series and considering each of the terms separately. We restrict ourselves to the leading harmonic in this expansion since at late times the unharmonicity is small.

(iii) The cumulants contain different combinations of $a_{k}$ and $a_{k}^{*}$, see Eqs. (B4). It is well known that the leading contribution to the resulting kinetic equation is due to the "resonant wave interactions," or, in the language of particle physics, only those terms survive, which are on the "mass shell." In our case those will be $\left\langle a_{\boldsymbol{p}}^{*} a_{\boldsymbol{p}_{1}} a_{\boldsymbol{p}_{2}}\right\rangle_{c}$ and $\left\langle a_{\boldsymbol{p}}^{*} a_{\boldsymbol{p}_{1}}^{*} a_{\boldsymbol{p}_{2}}\right\rangle_{c}$ for interactions which involve the zero mode, and $\left\langle a_{\boldsymbol{p}}^{*} a_{\boldsymbol{p}_{1}}^{*} a_{\boldsymbol{p}_{2}} a_{\boldsymbol{p}_{3}}\right\rangle_{c}$ otherwise. We restrict our attention to these cumulants only.

(iv) We neglect "anomalous" correlators, $\sigma_{k}$. These are small in the inertial range of turbulence as our lattice calculations show, but may be important otherwise.

\section{Leading asymptotic of collision terms in kinetic approximation}

For a free random field the cumulants Eqs. (B23) and (B24) are zero, and $\dot{n}_{k}=0$ to the first order in perturbation theory. To calculate $\dot{n}_{k}$ in second order one has to know the solutions for cumulants in the first order with respect to interactions.

We use the equation of motion for wave amplitudes, Eq. (B20), to calculate the time derivatives of the cumulants, $\partial_{t} a_{c}^{*}\left\langle a_{\boldsymbol{p}}^{*} a_{\boldsymbol{p}_{1}} a_{\boldsymbol{p}_{2}}\right\rangle_{c}$ and $\partial_{t}\left\langle a_{\boldsymbol{p}}^{*} a_{\boldsymbol{p}_{1}}^{*} a_{\boldsymbol{p}_{2}} a_{\boldsymbol{p}_{3}}\right\rangle_{c}$. Higher order correlators which appear in this procedure can be used in zeroth order of perturbation theory, i.e. they can be decomposed in $n_{k}$ assuming Gaussianity. To simplify the equations we use the following notations for products of $n_{k}$ which appear in these decompositions

$$
\begin{aligned}
& \mathrm{F}_{\boldsymbol{p}_{1} \boldsymbol{p}_{2}}^{\boldsymbol{p}} \equiv n_{c} n_{\boldsymbol{p}_{1}} n_{\boldsymbol{p}_{2}}-n_{c} n_{\boldsymbol{p}}\left(n_{\boldsymbol{p}_{1}}+n_{\boldsymbol{p}_{2}}\right), \\
& \mathrm{F}_{\boldsymbol{p}_{2} \boldsymbol{p}_{3}}^{\boldsymbol{p} \boldsymbol{p}_{1}} \equiv\left(n_{\boldsymbol{p}}+n_{\boldsymbol{p}_{1}}\right) n_{\boldsymbol{p}_{2}} n_{\boldsymbol{p}_{3}}-n_{\boldsymbol{p}} n_{\boldsymbol{p}_{1}}\left(n_{\boldsymbol{p}_{2}}+n_{\boldsymbol{p}_{3}}\right) .
\end{aligned}
$$


We find, keeping the terms which will have resonant behavior

$$
\begin{aligned}
\partial_{t} a_{c}^{*}\left\langle a_{\boldsymbol{p}}^{*} a_{p_{1}} a_{p_{2}}\right\rangle_{c} \simeq & i\left(\omega_{c}+\omega_{p}-\omega_{p_{1}}-\omega_{p_{2}}\right) a_{c}^{*}\left\langle a_{\boldsymbol{p}}^{*} a_{p_{1}} a_{p_{2}}\right\rangle_{c} \\
& +\frac{i 6 \lambda \delta^{(d)}\left(\boldsymbol{p}-\boldsymbol{p}_{1}-\boldsymbol{p}_{2}\right)}{(2 \pi)^{d / 2} \sqrt{2 \omega_{c} 2 \omega_{p} 2 \omega_{p_{1}} 2 \omega_{p_{2}}}} \mathrm{~F}_{p_{1} p_{2}}^{p}, \\
\partial_{t}\left\langle a_{\boldsymbol{p}}^{*} a_{\boldsymbol{p}_{1}}^{*} a_{p_{2}} a_{\boldsymbol{p}_{3}}\right\rangle_{c} \simeq & i\left(\omega_{p}+\omega_{p_{1}}-\omega_{p_{2}}-\omega_{p_{3}}\right)\left\langle a_{\boldsymbol{p}}^{*} a_{\boldsymbol{p}_{1}}^{*} a_{\boldsymbol{p}_{2}} a_{p_{3}}\right\rangle_{c} \\
& +\frac{i 6 \lambda \delta^{(d)}\left(\boldsymbol{p}+\boldsymbol{p}_{1}-\boldsymbol{p}_{2}-\boldsymbol{p}_{3}\right)}{(2 \pi)^{3 d / 2} \sqrt{2 \omega_{p} 2 \omega_{p_{1}} 2 \omega_{p_{2}} 2 \omega_{p_{3}}}} \mathrm{~F}_{p_{2} p_{3}}^{p p_{1}} .
\end{aligned}
$$

These equations have the common structure, $i \dot{J}=\Delta \omega J-A$. Since $A$ corresponds to the zeroth order in perturbations, it assumed to be time independent here. An appropriate particular solution for cumulants is therefore given by $J$ $=A /(\Delta \omega+i \epsilon)$, see e.g. Ref. [69]. Using the relation $\operatorname{Im}(x$ $+i \epsilon)^{-1}=-\pi \delta(x)$ we obtain

$$
\begin{aligned}
& \operatorname{Im} a_{c}^{*}\left\langle a_{\boldsymbol{p}}^{*} a_{\boldsymbol{p}_{1}} a_{\boldsymbol{p}_{2}}\right\rangle_{c} \\
& \simeq 3 \lambda \frac{\delta^{(d)}\left(\boldsymbol{p}-\boldsymbol{p}_{1}-\boldsymbol{p}_{2}\right) \delta\left(\omega_{c}+\omega_{p}-\omega_{p_{1}}-\omega_{p_{2}}\right)}{(2 \pi)^{d / 2-1} \sqrt{2 \omega_{c} 2 \omega_{p} 2 \omega_{p_{1}} 2 \omega_{p_{2}}}} \\
& \times \mathrm{F}_{\boldsymbol{p}_{1} \boldsymbol{p}_{2}}^{\boldsymbol{p}}, \\
& \operatorname{Im}\left\langle a_{\boldsymbol{p}}^{*} a_{\boldsymbol{p}_{1}}^{*} a_{\boldsymbol{p}_{2}} a_{\boldsymbol{p}_{3}}\right\rangle_{c} \\
& \simeq 3 \lambda \frac{\delta^{(d)}\left(\boldsymbol{p}+\boldsymbol{p}_{1}-\boldsymbol{p}_{2}-\boldsymbol{p}_{3}\right) \delta\left(\omega_{p}+\omega_{p_{1}}-\omega_{p_{2}}-\omega_{p_{3}}\right)}{(2 \pi)^{3 d / 2-1} \sqrt{2 \omega_{p} 2 \omega_{p_{1}} 2 \omega_{p_{2}} 2 \omega_{p_{3}}}} \\
& \times \mathrm{F}_{\boldsymbol{p}_{2} \boldsymbol{p}_{3}}^{\boldsymbol{p}_{\boldsymbol{p}_{1}}}
\end{aligned}
$$

\section{Isotropic wave kinetic equations}

Applying this result to Eqs. (B23) and (B24) we obtain the kinetic equation for wave occupation numbers $n_{k}$,

$$
\dot{n}_{k}=I_{k}^{(3)}+I_{k}^{(4)},
$$

where

$$
\begin{aligned}
& I_{k}^{(3)}=\int d \Omega{ }_{p_{1} p_{2}}^{k} \mathrm{~F}_{p_{1} p_{2}}^{k}-2 \int d \Omega{ }_{p_{2}}^{k p_{1}} \mathrm{~F}_{k p_{1}}^{p_{2}}, \\
& I_{k}^{(4)}=\int d \Omega_{p_{2} p_{3}}^{k p_{1}} \mathrm{~F}_{p_{2} p_{3}}^{k p_{1}}
\end{aligned}
$$

and

$$
\begin{gathered}
d \Omega_{p_{1} p_{2}}^{k} \equiv \frac{18 \lambda^{2} d^{d} p_{1} d^{d} p_{2} \delta^{(d)}\left(\boldsymbol{k}-\boldsymbol{p}_{1}-\boldsymbol{p}_{2}\right)}{(2 \pi)^{d-1} 2 \omega_{c} 2 \omega_{k} 2 \omega_{p_{1}} 2 \omega_{p_{2}}} \\
\times \delta\left(\omega_{c}+\omega_{k}-\omega_{p_{1}}-\omega_{p_{2}}\right), \\
d \Omega_{p_{2}}^{k p_{1}}:=\frac{18 \lambda^{2} d^{d} p_{1} d^{d} p_{2} \delta^{(d)}\left(\boldsymbol{k}+\boldsymbol{p}_{1}-\boldsymbol{p}_{2}\right)}{(2 \pi)^{d-1} 2 \omega_{k} 2 \omega_{p_{1}} 2 \omega_{p_{2}} 2 \omega_{c}} \\
\quad \times \delta\left(\omega_{k}+\omega_{p_{1}}-\omega_{p_{2}}-\omega_{c}\right), \\
d \Omega_{p_{2} p_{3}}^{k p_{1}} \equiv \frac{18 \lambda^{2} d^{d} p_{1} d^{d} p_{2} d^{d} p_{3} \delta^{(d)}\left(\boldsymbol{k}+\boldsymbol{p}_{1}-\boldsymbol{p}_{2}-\boldsymbol{p}_{3}\right)}{(2 \pi)^{2 d-1} 2 \omega_{k} 2 \omega_{p_{1}} 2 \omega_{p_{2}} 2 \omega_{p_{3}}} \\
\times \delta\left(\omega_{k}+\omega_{p_{1}}-\omega_{p_{2}}-\omega_{p_{3}}\right) .
\end{gathered}
$$

Both terms in Eq. (B31) describe scattering processes of two waves into two other ones. In Eq. (B32) one of them comes from the zero mode, while in Eq. (B33) all four are from the fluctuation field. Energy conservation in the interactions with the zero mode, $\omega_{c}+\omega_{k}-\omega_{p_{1}}-\omega_{p_{2}}=\varepsilon_{k}-\varepsilon_{p_{1}}$ $-\varepsilon_{p_{2}}$, can be written as conservation of the energies

$$
\varepsilon_{p} \equiv \omega_{p}-\omega_{c}
$$

which for small zero-mode amplitude (where $\omega_{c} \simeq \omega_{p=0}$ ) equals to the relativistic kinetic energy. Therefore, transport of energy over momentum space should be considered as transport of kinetic energy in this case.

These relations, Eqs. (B32) and (B33), for 3- and 4-particle collision integrals can be reduced to one and two dimensional integrations respectively, if the distribution functions are isotropic, for details see e.g. Refs. $[69,85]$.

The collision integral for 3-particle interactions in $d=3$ takes the form

$$
\begin{aligned}
I_{k}^{(3)}= & \frac{9 \lambda^{2} \bar{\phi}_{0}^{2}}{16 \pi \omega_{k} k}\left(\int_{0}^{\varepsilon_{k}} d \varepsilon_{2}\left[n_{3} n_{2}-n_{k}\left(n_{3}+n_{2}\right)\right]\right. \\
& \left.+2 \int_{\varepsilon_{k}}^{\infty} d \varepsilon_{2}\left[n_{2}\left(n_{k}+n_{1}\right)-n_{k} n_{1}\right]\right)
\end{aligned}
$$

where $\varepsilon_{i} \equiv \varepsilon\left(p_{i}\right)$ and $n_{i} \equiv n\left(\varepsilon_{i}\right)$. Energy conservation in this case corresponds to $\varepsilon_{3}=\varepsilon_{k}-\varepsilon_{2}$ and $\varepsilon_{1}=\varepsilon_{2}-\varepsilon_{k}$.

The collision integral for 4-particle interactions in $\lambda \phi^{4}$ theory reduces to

$$
I_{k}^{(4)}=\frac{9 \lambda^{2}}{32 \pi^{3} \omega_{k} k} \int_{0}^{\infty} d \varepsilon_{2} \int_{0}^{\infty} d \varepsilon_{3} D F(n),
$$

where $D \equiv \min \left[k, p_{1}, p_{2}, p_{3}\right]$ and $\varepsilon_{1}=\varepsilon_{2}+\varepsilon_{3}-\varepsilon_{k}>0$ in arguments of $F(n)=\left(n_{k}+n_{1}\right) n_{2} n_{3}-n_{k} n_{1}\left(n_{2}+n_{3}\right)$.

Note an interesting fact: Apart of the prefactor, Eqs. (B39) and (B38) are functions of relativistic kinetic energy, $I_{k}^{(i)}$ $\equiv\left(\omega_{k} k\right)^{-1} f\left(\varepsilon_{k}\right)$. This gives for the flux of kinetic energy in 3 dimensions [cf. with Eq. (15)] 


$$
\begin{aligned}
S^{\rho}(k) & =-\int^{k} \frac{d^{3} p}{(2 \pi)^{3}} \varepsilon_{p} I_{p}^{(3)} \\
& =-\frac{1}{2 \pi^{2}} \int^{\varepsilon_{k}} d \varepsilon \varepsilon f(\varepsilon),
\end{aligned}
$$

where we have used $p d p=\omega d \varepsilon$. Therefore, the turbulent flux should correspond to a particle distribution being a power law of relativistic kinetic energy. Remarkably, we have solved for the turbulent fluxes without the usual assumption of scale-independent dispersion law, Eq. (17). In fact, the dispersion law was that of relativistic field theory, $\omega_{k}^{2}=k^{2}+M_{\text {eff }}^{2}$. We do observe a single power law for the particle distributions as functions of kinetic energy in our lattice integration, even in situations when $M_{\text {eff }}^{2}$ is large in the inertial range, see Fig. 4, the upper panel.

\section{Kinetic equation for zero mode}

The kinetic equation for the wave occupation numbers has to be supplemented by the kinetic equation for the zero mode. We start with the equation

$$
\ddot{\phi}_{0}+\omega_{c}^{2} \phi_{0}=-\lambda\left\langle\delta \phi^{3}\right\rangle
$$

and repeat the procedure of the previous subsections. As ana$\log$ of Eq. (B21) we obtain

$$
\dot{n}_{c}=2 \lambda \operatorname{Im}\left(\frac{a_{c}^{*}}{\sqrt{2 \omega_{c}}}\left\langle\delta \phi^{3}\right\rangle\right) .
$$

Substituting Eq. (B4) and solving equation for higher order correlators we get

$$
\dot{n}_{c}=-\int \frac{d^{d} k}{(2 \pi)^{d}} I_{k}^{(3)} .
$$

This result is not surprising since 4-particle collisions conserve particle number. In the model we consider, 3-particle interactions are derived from the 4-particle collisions with one of the particles being replaced by the condensate. Therefore, Eq. (B43) can be interpreted as a conservation of the total occupation number, in particles and in the condensate, $n_{c}+(2 \pi)^{-d} \int d^{d} k n_{k}=$ const.
[1] A.D. Linde, Particle Physics and Inflationary Cosmology (Harwood Academic, New York, 1990).

[2] E.W. Kolb and M.S. Turner, The Early Universe (AddisonWesley, Reading, MA, 1990).

[3] A.R. Liddle and D.H. Lyth, Cosmological Inflation and LargeScale Structure (Cambridge University Press, Cambridge, England, 2000).

[4] L. Kofman, A.D. Linde, and A.A. Starobinsky, Phys. Rev. Lett. 73, 3195 (1994).

[5] J.H. Traschen and R.H. Brandenberger, Phys. Rev. D 42, 2491 (1990).

[6] Y. Shtanov, J. Traschen, and R.H. Brandenberger, Phys. Rev. D 51, 5438 (1995).

[7] S.Y. Khlebnikov and I.I. Tkachev, Phys. Rev. Lett. 77, 219 (1996).

[8] S.Y. Khlebnikov and I.I. Tkachev, Phys. Lett. B 390, 80 (1997).

[9] S.Y. Khlebnikov and I.I. Tkachev, Phys. Rev. Lett. 79, 1607 (1997).

[10] T. Prokopec and T.G. Roos, Phys. Rev. D 55, 3768 (1997).

[11] L. Kofman, A.D. Linde, and A.A. Starobinsky, Phys. Rev. D 56, 3258 (1997).

[12] J. Garcia-Bellido and A.D. Linde, Phys. Rev. D 57, 6075 (1998).

[13] G.N. Felder et al., Phys. Rev. Lett. 87, 011601 (2001).

[14] G.F. Giudice, M. Peloso, A. Riotto, and I. Tkachev, J. High Energy Phys. 08, 014 (1999).

[15] L. Kofman, A.D. Linde, and A.A. Starobinsky, Phys. Rev. Lett. 76, 1011 (1996).

[16] I.I. Tkachev, Phys. Lett. B 376, 35 (1996).

[17] A. Riotto and I.I. Tkachev, Phys. Lett. B 385, 57 (1996).

[18] S. Khlebnikov, L. Kofman, A.D. Linde, and I.I. Tkachev, Phys. Rev. Lett. 81, 2012 (1998).
[19] I.I. Tkachev, S. Khlebnikov, L. Kofman, and A.D. Linde, Phys. Lett. B 440, 262 (1998).

[20] S. Kasuya and M. Kawasaki, Phys. Rev. D 56, 7597 (1997).

[21] A. Rajantie and E.J. Copeland, Phys. Rev. Lett. 85, 916 (2000).

[22] R. Ray and S. Sengupta, Phys. Rev. D 65, 063521 (2002).

[23] E.J. Copeland, S. Pascoli, and A. Rajantie, Phys. Rev. D 65, 103517 (2002).

[24] G.N. Felder, L. Kofman, and A.D. Linde, Phys. Rev. D 59, 123523 (1999).

[25] S.Y. Khlebnikov and I.I. Tkachev, Phys. Rev. D 56, 653 (1997).

[26] E.W. Kolb, A.D. Linde, and A. Riotto, Phys. Rev. Lett. 77, 4290 (1996).

[27] E.W. Kolb, A. Riotto, and I.I. Tkachev, Phys. Lett. B 423, 348 (1998).

[28] J. Garcia-Bellido, D.Y. Grigoriev, A. Kusenko, and M.E. Shaposhnikov, Phys. Rev. D 60, 123504 (1999).

[29] J. Garcia-Bellido and E. Ruiz Morales, Phys. Lett. B 536, 193 (2002).

[30] J.R. Ellis, A.D. Linde, and D.V. Nanopoulos, Phys. Lett. 118B, 59 (1982).

[31] J.R. Ellis, D.V. Nanopoulos, and S. Sarkar, Nucl. Phys. B259, 175 (1985).

[32] R. Kallosh, L. Kofman, A.D. Linde, and A. Van Proeyen, Phys. Rev. D 61, 103503 (2000).

[33] G.F. Giudice, I. Tkachev, and A. Riotto, J. High Energy Phys. 08, 009 (1999).

[34] G.F. Giudice, A. Riotto, and I. Tkachev, J. High Energy Phys. 11, 036 (1999).

[35] A.L. Maroto and A. Mazumdar, Phys. Rev. Lett. 84, 1655 (2000). 
[36] D.J.H. Chung, E.W. Kolb, and A. Riotto, Phys. Rev. D 59, 023501 (1999).

[37] V. Kuzmin and I.I. Tkachev, JETP Lett. 68, 271 (1998), [Pisma Zh. Éksp. Teor. Fiz 68, 255 (1998)].

[38] V. Kuzmin and I.I. Tkachev, Phys. Rev. D 59, 123006 (1999).

[39] D.J.H. Chung, E.W. Kolb, A. Riotto, and I.I. Tkachev, Phys. Rev. D 62, 043508 (2000).

[40] L. Covi, L. Roszkowski, R.R. de Austri, and M. Small, J. High Energy Phys. 0406, 003 (2004).

[41] A.R. Liddle and D.H. Lyth, Phys. Rep. 231, 1 (1993).

[42] S. Dodelson and L. Hui, Phys. Rev. Lett. 91, 131301 (2003).

[43] A.R. Liddle and S.M. Leach, Phys. Rev. D 68, 103503 (2003).

[44] B. Feng, X. Gong, and X. Wang, astro-ph/0301111.

[45] T. Hamazaki and H. Kodama, Prog. Theor. Phys. 96, 1123 (1996)

[46] G. Dvali, A. Gruzinov, and M. Zaldarriaga, Phys. Rev. D 69, 023505 (2004).

[47] L. Kofman, astro-ph/0303614.

[48] K. Enqvist, A. Mazumdar, and M. Postma, Phys. Rev. D 67, 121303 (2003).

[49] S. Matarrese and A. Riotto, J. Cosmol. Astropart. Phys. 0308, 007 (2003).

[50] K.A. Olive, G. Steigman, and T.P. Walker, Phys. Rep. 333, 389 (2000).

[51] D.T. Son, Phys. Rev. D 54, 3745 (1996).

[52] D.V. Semikoz, Helv. Phys. Acta 69, 207 (1996).

[53] G. Aarts, G.F. Bonini, and C. Wetterich, Nucl. Phys. B587, 403 (2000).

[54] S. Davidson and S. Sarkar, J. High Energy Phys. 11, 012 (2000).

[55] M. Salle, J. Smit, and J.C. Vink, Phys. Rev. D 64, 025016 (2001).

[56] E. Calzetta and M. Thibeault, Phys. Rev. D 63, 103507 (2001).

[57] S. Borsanyi, A. Patkos, J. Polonyi, and Z. Szep, Phys. Rev. D 62, 085013 (2000).

[58] G.N. Felder and L. Kofman, Phys. Rev. D 63, 103503 (2001).

[59] S. Borsanyi and Z. Szep, Phys. Lett. B 508, 109 (2001).

[60] D. Bodeker, Nucl. Phys. B, Proc. Suppl. 94, 61 (2001).

[61] G. Aarts, D. Ahrensmeier, R. Baier, J. Berges, and J. Serreau, Phys. Rev. D 66, 045008 (2002).

[62] J. Berges and J. Serreau, Phys. Rev. Lett. 91, 111601 (2003).

[63] S. Borsanyi, A. Patkos, and D. Sexty, Phys. Rev. D 68, 063512 (2003).

[64] D. Boyanovsky, C. Destri, and H.J. de Vega, Phys. Rev. D 69, 045003 (2004).

[65] J. Baacke and A. Heinen, Phys. Rev. D 69, 083523 (2004).

[66] T. Ikeda, Phys. Rev. D 69, 105018 (2004).
[67] R. Micha and I.I. Tkachev, Phys. Rev. Lett. 90, 121301 (2003).

[68] Michael G. Schmidt, Proceedings on the Conference on Strong and Electroweak Matter (SEWM 2002), Heidelberg, Germany, 2002.

[69] V. Zakharov, V. L'vov, and G. Falkovich, Kolmogorov Spectra of Turbulence, Wave Turbulence (Springer-Verlag, Berlin, 1992).

[70] V. L'vov, Wave Turbulence under Parametric Exitation (Springer-Verlag, Berlin, 1994).

[71] U. Frisch, Turbulence (Cambridge University Press, Cambridge, England, 1995).

[72] A.K. Kolmogorov, Dokl. Akad. Nauk SSSR 30, 301 (1941).

[73] A.K. Kolmogorov, Dokl. Akad. Nauk SSSR 31, 538 (1941).

[74] A.N. Pushkarev and V.E. Zakharov, J. Appl. Mech. Tech. Phys. 4, 506 (1967).

[75] A.D. Linde, Phys. Lett. 129B, 177 (1983).

[76] A.D. Linde, Phys. Rev. D 49, 748 (1994).

[77] R. Micha and M.G. Schmidt, Eur. Phys. J. C 14, 547 (2000).

[78] D.I. Kaiser, Phys. Rev. D 56, 706 (1997).

[79] P.B. Greene, L. Kofman, A.D. Linde, and A.A. Starobinsky, Phys. Rev. D 56, 6175 (1997).

[80] D.I. Kaiser, Phys. Rev. D 57, 702 (1998).

[81] V.E. Zakharov, S.L. Musher, and A.M. Rubenchik, Phys. Rep. 129, 285 (1985).

[82] A.C. Newell, S. Nazarenko, and L. Biven, Physica D 152/153, 520 (2001).

[83] A.V. Kats, Zh. Éksp. Teor. Fiz. 71, 2104 (1976), [Sov. Phys. JETP 44, 1106 (1976)].

[84] D.V. Semikoz and I.I. Tkachev, Phys. Rev. Lett. 74, 3093 (1995).

[85] D.V. Semikoz and I.I. Tkachev, Phys. Rev. D 55, 489 (1997).

[86] G. Falkovich and A. Shafarenko, J. Nonlinear Sci. 1, 457 (1991).

[87] S. Dyachenko, A. Newell, A. Pushkarev, and V. Zakharov, Physica D 57, 96 (1992).

[88] A.N. Pushkarev and V.E. Zakharov, Physica D 135, 98 (2000).

[89] I.I. Tkachev, Phys. Lett. B 261, 289 (1991).

[90] G.N. Felder and I.I. Tkachev, hep-ph/0011159.

[91] W.H. Press, B.P. Flannery, S.A. Teukolsky, and W.T. Vetterling, Numerical Recepies in C (Cambridge University Press, Cambridge, England, 1988).

[92] M. Abramowitz and I.A. Stegun, editors, Pocketbook of Mathematical Functions (Verlag Harri Deutsch, Thun and Frankfurt/Main, 1984).

[93] Higher Transcendental Functions, edited by A. Erdelyi (McGraw-Hill, New York, 1953), Vol. II.

[94] G. Parisi, Statistical Field Theory (Addison-Wesley, Redwood City, CA, 1988). 